The non-linear dynamics of bending instability and vertical structure of a galactic stellar disc embedded into a spherical halo are studied with N-body numerical modelling. Development of the bending instability in stellar galactic disc is considered as the main factor that increases the disc thickness. Correlation between the disc vertical scale height and the halo-to-disc mass ratio is predicted from the simulations. The method of assessment of the spherical-todisc mass ratio for edge-on spiral galaxies with a small bulge is considered. Modelling of eight edge-on galaxies: NGC 891, NGC 4738, NGC 5170, UGC 6080, UGC 7321, UGC 8286, UGC 9422 and UGC 9556 is performed. Parameters of stellar discs, dark haloes and bulges are estimated. The lower limit of the dark-toluminous mass ratio in our galaxies is of the order of 1 within the limits of their stellar discs. The dark haloes dominate by mass in the galaxies with very thin stellar discs (NGC 5170, UGC 7321 and UGC 8286).

Key words: galaxies: haloes - galaxies: spiral - galaxies: structure - dark matter 


\title{
Numerical modelling of the vertical structure and dark halo parameters in disc galaxies
}

\author{
A. Khoperskov ${ }^{1}$, D. Bizyaev ${ }^{2,3}$, N. Tiurina ${ }^{2}$, M. ButenKo $^{1}$ \\ (1) Volgograd State University, Volgograd, 400062, Russia \\ (2) Sternberg Astronomical Institute, Moscow, 119992, Russia \\ (3) Apache Point Observatory and NMSU, Sunspot, NM,USA
}

Accepted ???? ???? ??. Received ???? ???? ??; in original form ???? ???? ??

Abstract.

\section{Introduction}

The luminous matter reveals only a part of the total mass in galaxies. Additional fraction of non-luminous matter is required to explain rotation curves ( $\mathrm{RC}$ hereafter) of spiral galaxies. Fitting of galactic rotation curves does not strictly require a lot of dark matter. Obviously, a modest dark halo with $1 / 4$ of the disc's mass is enough to explain a flat RC at the radii range $r \simeq(2-4) \cdot L$ (van Albada et al. 1985), where $L$ is the disc scale length. Numerical modelling of disc galaxies without a massive spheroidal subsystem shows that their stellar discs heat up significantly during the evolution, and finally achieve the equilibrium state with too high velocity dispersion (Ostriker \& Peebles 1973, Carlberg \& Sellwood 1985,

Athanasoula \& Sellwood 1986.

Bottema \& Gerritsen 1997, Fuchs \& von Linden 1998

Khoperskov et al. 2003). To explain the low ratio of the stellar velocity dispersion to the circular velocity $c^{o b s} / V_{c}$, which is observed in many galaxies at $r>2 L$, a spherical subsystem as massive as the the stellar disc or even more has to be incorporated into the models. More additional arguments for the massive spherical haloes come from observations of galactic polar rings and from long flat RCs observed far outside the limits of stellar discs (Whitmore et al. 1987, Reshetnikov \& Sotnikova 2000, Iodice et al. 2003, Combes 2002).

Mass of the spherical subsystem in a galaxy can be determined from its rotation with quite high level of uncertainty. Incorporation of radial distribution of the stellar velocity dispersion into the modelling helps

Correspondence to: dmbiz@apo.nmsu.edu to decrease the ambiguities and allows to constrain better the range of parameters of galactic subsystems (Bottema 1993, Bottema \& Gerritsen 1997, Fux 1997. Khoperskov et al. 2001). Unfortunately, observations of the stellar velocity dispersion in galactic discs require a sensitive spectroscopy and long integration time, so the dispersion still can be estimated for a limited number of galaxies. An alternative method of including the velocity dispersion into the numerical modelling is to utilize the disc thickness that, in a contrary, is easy to observe in many edge-on galaxies. Relation between the vertical scale height, local disc surface density and spheroidal subsystem mass $M_{s}$ enables us to include the disc thickness into the modelling. At the same time, if the velocity dispersion is close to that required for the marginal disc stability, the thickness of the stellar disc is tightly connected with the spherical-to-disc components mass ratio (Zasov et al. 1991, Mikhailova et al. 2001, Sotnikova \& Rodionov 2006).

One of important problems in the physics of galaxies is the identification of the main factor that increases the random velocity from $6 \div 10 \mathrm{~km} / \mathrm{s}$ (young population) to the values of $\sim 20 \div 150 \mathrm{~km} / \mathrm{s}$ (observed in old stellar population). Besides such factors of heating as the scattering of stars by giant molecular clouds (Spitzer \& Schwarzschild 1951. Spitzer \& Schwarzschild 1953) and in the spiral arms (Jenkins \& Binney 1990), the bending instability is the most important mechanism of the vertical velocity dispersion $c_{z}$ growth in disc galaxies (Poliachenko \& Shukhman 1977, Zasov et al. 1991, Mikhailova et al. 2001, Sotnikova \& Rodionov 2003 Sotnikova \& Rodionov 2005). 
The bending instability in galactic discs has been considered with the help of N-body simulations. Essential results were obtained when the tidal interaction was taken into account (Hernquist et al. 1993. Weinberg 1998 Velazquez \& White 1999, Mayer et al. 2001. Bailin \& Steinmetz 2003). Tidal forces are generated by the disc bending and increase the disc thickness. This is in agreement with observations of the probability of bending and warps in isolated galaxies and group objects (Reshetnikov \& Combes 2002). The emergence of the bending instabilities was investigated by Raha et al. 1991, Sellwood 1996, Sellwood \& Merritt 1994, Patsis et al. 2002, Griv et al. 2002, Binney et al. 1998. A model of the galaxy NGC 2146 that involved the bending instability was considered by Griv et al. 1998. The bending instability reveals itself on much longer time scale, typically $10^{9}$ years (Sotnikova \& Rodionov 2003) in the comparison with the gravitational instability in the disc plane. Hunter \& Toomre 1969 explained the global warps observed in discs of some isolated galaxies by bending instabilities.

The disc scale height in galaxies depends on the vertical velocity dispersion $c_{z}(r)$. The latter is dependent of the radial velocity dispersion $c_{r}(r)$. A discussion about the relation between $c_{r}$ and $c_{z}$ was opened firstly by Toomre 1966. Considering a simplified model of infinitely thin uniform self-gravitating layer, (Poliachenko \& Shukhman 1977) found an essential condition for a stellar system stability against the small-scale bending perturbations: $c_{z} / c_{r} \geq 0.37$. The dynamics of the bending instabilities was also considered by Araki 1986, where a non-uniform volume density distribution in the $z$-direction was taken into account. The conclusion about a lower value of the critical ratio $c_{z} / c_{r} \gtrsim 0.3$ was made in that paper (see also discussion in Merrit \& Sellwood 1994).

In this paper we point our special attention to the radial distribution of the disc thickness and ratio $c_{z} / c_{r}$ that are necessary to provide the disc stability against different kinds of bending perturbations. We mostly consider isolated late type galaxies without a prominent bulge. The presence of bulge would complicate the analysis of bending instability. If there is no bulge in a galaxy, one can restore the internal part of rotation curve assuming that it is defined by the disc component only in the inner $(r \lesssim 2 L$ ) regions (Zasov \& Khoperskov 2003). However, we also develop our modelling for two wellstudied disc galaxies with significant bulge component because we can incorporate observations of of the stellar velocity dispersion for these galaxies.

In $\S 2$ we describe our N-body model. Evolution and features of the bending perturbations are considered in $\S 3$. In $\S 4$ we model structural parameters and rotation curves of eight edge-on galaxies and evaluate their best fitting parameters focusing at dark halo-to-disc mass ratio.

\section{Dynamical modelling of stellar discs in galaxies}

Our N-body modelling is based on the numerical integration of the motion equations for $N$ gravitationally interacting particles as it was described in detail by Khoperskov et al. 2003. This system of collisionless particles has a shape of a disc (and, optionally, bulge) embedded into a spherical dark halo. The halo and bulge are represented by fixed potentials. The steady state distribution of mass in the bulge $\varrho^{(b)}$ and halo $\varrho^{(h)}$ are defined as:

$\varrho^{(h, b)}(r)=\varrho_{0}^{(h, b)} \varnothing\left(1+\xi^{2} / a_{(h, b)}^{2}\right)^{k}$,

where $\xi=\sqrt{r^{2}+z^{2}}$, and $(r, z)$ are the radial and vertical coordinates, $k$ equals to $3 / 2$ for the bulge and to 1 for the halo. The dimensional spatial scales for the bulge and halo are denoted as $a_{b}$ and $a_{h}$, respectively. Our model bulge is defined within a sphere with radius $\xi \leq r_{b}^{\max }$.

The initial vertical equilibrium in the disc is defined by the Poisson equation

$\partial \varnothing r \partial r(r \partial \Phi \emptyset \partial r)+\partial^{2} \Phi \varnothing \partial z^{2}=4 \pi G\left(\varrho+\varrho^{h}+\varrho^{b}\right)$

and by a balance of forces in the vertical direction (in the first-order approximation)

$c_{z}^{2} \partial \varrho \varnothing \partial z=-\varrho \partial \Phi \varnothing \partial z$.

Here $\Phi$ is the gravitational potential, $\varrho$ is the disc volume density and $c_{z}$ is the vertical component of velocity dispersion. The radial component of Jeans equation defines the rotational velocity in the stellar disc (Valluri 1994):

$V^{2}=V_{c}^{2}+c_{r}^{2}\left\{1-c_{\varphi}^{2} \phi c_{r}^{2}+r \varnothing \varrho c_{r}^{2} \partial\left(\varrho c_{r}^{2}\right) \emptyset \partial r+r \varnothing c_{r}^{2} \partial\langle u w\rangle \varnothing \partial z\right\}$,

where the brackets $\langle\ldots\rangle$ denote the mean value. The last term in the figured brackets in (4) is the chaotic part of the radial $u$ and vertical $w$ velocity components.

The system of equations (2)-(3) can be reduced to the equation for dimensionless disc density $f(z ; r)=$ $\varrho(z ; r) / \varrho(z=0 ; r)$, see Bahcall 1984.

$d^{2} f \varnothing d z^{2}+2 d \ln c_{z} \varnothing d z d f \varnothing d z-1 \varnothing f(d f \varnothing d z)^{2}+4 \pi G \emptyset c_{z}^{2} \sigma \varnothing 2 z_{0} \times$ $\times f\left(f+2 \varrho_{h}^{e f f} \emptyset \sigma z_{0}\right)=0$.

where the surface density is $\sigma=2 \varrho(z=0) \cdot z_{0}, \varrho_{h}^{\text {eff }}=$ $\varrho_{h}-1 \varnothing 4 \pi G r \partial V_{c}^{2} \varnothing \partial r$ and the disc scale height is $z_{0}=$ $\int_{0}^{\infty} f d z$. For the case of $c_{z}=$ const and $\varrho_{h}^{\text {eff }}=0$ the volume density profile of the disc in its vertical direction is:

$\varrho(z)=\varrho(z=0) \cdot \operatorname{sech}^{2}\left(z / z_{0}\right)$,

where the vertical disc scale for this case is $z_{0}=$ $c_{z}^{2} / \pi G \sigma$.

At the flat part of RC, the last term of equation (5) decreases the vertical scale $z_{0}$ because of additional gravitational potential. Nevertheless, this factor is not dominant and the most significant reason for $z_{0}$ decreasing is that the potential of the halo changes the value of 
$c_{z} / c_{r}$ required for the marginal stability of bending perturbations.

We assume that the disc volume density can be written

$\varrho(r, z)=\varrho(0,0) f_{r}(r) f_{z}(z)$

where the function $f_{z}(z)$ describes the vertical density profile. We consider two functional forms for the vertical density distribution $f_{z}(z)$ in our modelling: $\exp \left(-z / h_{z}\right)$ and $\operatorname{sech}^{2}\left(z / z_{0}\right)$, where the scale heights $h_{z}$ and $z_{0}$ are functions of time and location in the disc. The radial density profile $f_{r}(r)$ is assumed to be exponential: $f_{r}(r)=\exp (-r / L)$.

The mass $M_{h}$ of dark halo was calculated inside the maximum disc radius $R_{\max }$. We assume that $R_{\max } \approx 4 L$ in our modelling (in accordance to van der Kruit \& Searle 1981, van der Kruit \& Searle 1982, Pohlen et al. 2002, Holley-Bockelmann \& Mihos 2001). We also designate the spherical-to-disc mass ratio in the galaxies as $\mu$.

As the initial templates in our models, we consider axisymmetric systems in the state of equilibrium. Initial radial distributions of the velocity dispersions in radial $\left(c_{r}\right)$ and azimuthal $\left(c_{\varphi}=c_{r} \propto / 2 \Omega\right)$ directions keep the templates in gravitationally stable state. We assume that the stellar disc population is shaped as a quasi-stationary balanced system and that the radial and vertical random motions of particles keep the disc in the marginal stability against bending waves and perturbations in the disc plane. Then we observe the evolution of the discs during 10-40 turns at its outer edge.

We use TREE-code approximation in the modelling (described in detail by Khoperskov et al. 2003) with $N=(0.5-8) 10^{6}$. Also, in $\S 3.1 .1$ we verify some model conclusions with independent direct particleparticle approach (with less number of $\mathrm{N}$ ) as well described by Khoperskov et al. 2003

For the comparison between the modelling and observations, we incorporate both structural parameters of stellar discs and their rotation curves at the same time. We take into account that the observed structural parameters in edge-on galaxies are results of integration along the line of sight. To obtain the edge-on surface density, we integrate the volume density of the model disc along the line of sight:

$\sigma(x, z)=\int_{-y_{0}}^{y_{0}} \varrho(\xi, z) d y \quad\left(\xi=\sqrt{x^{2}+y^{2}}\right)$.

Here $x$ is the projected distance to the centre of the disc, $y_{0}=\sqrt{R_{\max }^{2}-x^{2}}$.

The similar approach is applied for the integration of RC along the line of sight (it was described by Zasov \& Khoperskov 2003).

\section{Bending instabilities in stellar discs}

\subsection{The global bending instability}

The linear analysis of stability against the global bending perturbations was discussed by Polyachenko \& Shukhman 1979 and generalized by Vandervoort 1991. It was shown that the large scale instability modes with the azimuthal numbers $m=1,2$ and 3 generate the instability in collisionless ellipsoidal systems in dependence of their axes ratio, velocity dispersion and mass of their spherical subsystems. The most important result from the papers mentioned above is that the area of stability in the parametric space depends on the spherical subsystem mass. Once the halo is massive enough, the systems with low velocity dispersion can be unstable. This gives an example of destabilizing role of the halo. On the other hand, the higher mass of the spherical subsystem requires the lower ratio of $c_{r} / V^{\max }$ that is necessary to keep the disc gravitationally stable. As a result, the destabilizing role of the massive halo related to the large scale bending perturbations may not reveal itself (Zasov et al. 1991, Mikhailova et al. 2001).

Our model stellar discs at the initial moment $(t=0)$ are axisymmetric, in the equilibrium state along both the radial and vertical directions and gravitationally stable in the disc plane. The latter is made by assigning a high value to the radial velocity dispersion $c_{r} \gtrsim(1.5 \div 4) \cdot c_{T}$, where the Toomre stability parameter $Q_{T}=c_{r} / c_{T}$ is a function of $r$ (Khoperskov et al. 2003, ?). In general, our initial disc templates can be either stable or unstable against the bending perturbations in dependence of $c_{z}(r)$ distribution.

The evolution of systems with a small initial value of $c_{z} / c_{r}$ (i.e. systems that are dynamically cold in the vertical direction) reveals a gradual growth of the global bending instability, which heats up the disc in the vertical direction and increases its thickness.

\subsubsection{The axisymmetric bending mode $(m=0)$}

Development of unstable axisymmetric bending mode $m=0$ leads to significant disc heating in the vertical direction (the same conclusion has been made by Sellwood 1996). The evolution of the vertical coordinate of the local centre of mass $\zeta(r, t)$, vertical velocity dispersion $c_{z}(r, t)$, disc scale height $z_{0}(r, t)$, dispersion ratio $\alpha_{z} \equiv c_{z}(r, t) / c_{r}(r, t)$, radial velocity dispersion $c_{r}(r, t)$ and rotational velocity $V(r, t)$ in the stellar disc where the axisymmetric mode is developing can be seen in Fig. 1. We fix the units hereafter assuming that $M_{d}=1, G=1$ and $4 L=1$. In this case the circular velocity $V_{c} \sim 1.5$ at the periphery of the disc and the corresponding dimensionless rotation period is $\tau \approx 4$.

As it is seen in Fig. 1 parameters of the disc stay almost unchanged for the first 2.5 turns $(t \approx 10$ in our units). This time the instability modes are being developed at the linear stage of the bending instability evo- 
lution. After $t \gtrsim 10$, the linear stage is superseded by the non-linear development of the bending instability, and the centre of mass $\zeta(r)$ oscillates with larger amplitude in the $z$-direction (see Fig. 11). The amplitude $\zeta(r)$ rapidly increases in the central regions of the disc up to its maximum value and then decreases down to the initial state (see curves 1-5 in Fig. 19), whereas the increasing of $\zeta(r)$ occurs much slower at the outer parts of the disc (curves 11-19). The rapid growth of the velocity dispersion $c_{z}$ and scale height $z_{0}$ begins after a time delay from $\zeta(r)$, see Fig.1b and c. A flare of stellar disc emerges in its central region and then propagates outside, towards the periphery. The value of $c_{z} / c_{r}$ rises mostly because of $c_{z}$ growth, and in less power due to $c_{r}$ decreasing (Fig. $1 \mathrm{~d}$ and e). The azimuthal component of the velocity dispersion $c_{\varphi}$ follows $c_{r}$, so the relation $c_{\varphi} \simeq c_{r} \varkappa / 2 \Omega$ is valid almost everywhere. This equation can be failed either in a very thick disc $\left(z_{0} / L \gtrsim 0.4\right)$, or in the very central region, where $c_{r} / c_{\varphi}<2 \Omega / \Re$, that means the anisotropy is lower in those regions because of the system spherization. Decreasing of $c_{r}$ and $c_{\varphi}$ is the result of conversion of the kinetic energy of random motions in the disc plane to the kinetic energy of random vertical motions. Note that development of this bending mode takes place in the disc which is initially axisymmetric over all parameters.

The value of $\zeta$ has opposite signs at the disc centre and periphery in the considering model (see Fig. 1a). This generates a "mexican hat-like" structure as a result of this instability (Fig. 2). The isolines of $\zeta$ have concentric shape. The intermediate regions and periphery at the moment $t=25.4 \simeq 6 \tau$ are shifted off the disc plane toward the opposite directions, whereas the central region $(r \lesssim 0.4$ ) has returned to its initial position, see Fig. 2b. The disc vertical structure driven by the bending mode $m=0$ is shown in Fig. 2 d. We can see a formation of box-shaped features in the disc, which is typical for all moments of evolution when the axisymmetric bending mode dominates. Note that this feature is not relevant to a bar. The considered instability may be responsible for the formation of central thickening of discs (i.e. bulges), and for the structure of S0 galaxies (see Fig. 2 $\mathrm{d}$ )).

A gradual change of the velocity dispersions $c_{z}$ and $c_{r}$ and rotational velocity $V$ begins when the amplitude of the vertical oscillations $\zeta$ grows significantly. The vertical disc heating is accompanied by the increasing of $c_{z} / c_{r}$, but favourable conditions for developing the bending instability disappear at certain values of $c_{z} / c_{r}$. As a result, new stable and thick disc forms. The specific time for this process depends significantly on the model parameters and is of the order of ten turns of the disc's outer regions. If the initial distribution of $\alpha_{z}=c_{z} / c_{r}$ was subcritical, the linear stage takes longer time due to low increment of instability, and because of the heating at the non-linear stage was feeble. The amplitude of the bending mode, and even the ability of its emergence, depends significantly on the initial radial distribution of $c_{z} / c_{r}$.
An important consequence is that the final distribution of $\alpha_{z}(r)$, as a result of bending instability, depends on its initial value $\alpha_{z}(r, t=0)$. After development of the global bending instability, those thin discs that had a low value of the scale height ratio gain larger ratio $c_{z} / c_{r}$ than it would required to keep the discs stable. The explanation can be found in the essentially non-linear nature of the disc heating. As a result, the parameter $c_{z} / c_{r}$ "jumps over" the "border of stability" and comes to relaxation well above its marginal threshold.

We verified the numerical effects of our TREEcode by reproducing the most important numerical experiments with a direct particle-particle (PP hereafter) code, see description by Khoperskov et al. 2003. The analogue of Fig. 1 1 that was obtained with the help of PP code that started from the same initial conditions and was evaluated with only $5 \times 10^{4}$ particles is shown in Fig. 3. The residuals in Fig. 3 are greater than those in Fig. 1 due to less number of particles. Nevertheless, all features from Fig. 1 can be seen in Fig. 3

If the model disc is not massive enough to provide the gravitational stability against the bar-mode and mass of the halo is low $(\mu \lesssim 1)$, the main reason for heating the initially thin cold disc is the axisymmetric bending mode ( $m=0$ ) whereas the modes $m=1,2$ do not emerge at all. Note that the mode $m=0$ may emerge far from the centre $(r \gtrsim L)$ and develop there without penetrating into the central part of the disc. It can happen because of two reasons: either the initial disc had $c_{z} / c_{r} \geq \alpha_{z}^{\text {crit }}$ (see $\S 3.4$ ) and was stable in the central regions whereas periphery of the disc is thin and unstable (Fig. 4), or the galaxy harbours a concentrated and massive bulge. In such models the bending modes in the centre have low amplitude. The presence of a bulge and/or a halo plays a stabilizing role. Hence, having all other conditions equal, the discs of galaxies possessing bulges have to look thinner. Note that since we did not consider dynamically non-stationary bulges in our model, this our conclusion requires further investigation.

\subsubsection{Bending modes $m=1,2$}

In the models with massive halo $(\mu \gtrsim 2)$, the axisymmetric bending modes $m=1$ and $m=2$ may develop themselves and heat up the stellar disc along its vertical direction. The surface density of the disc remains axisymmetric. The evolution of initially thin disc for a case of a heavy halo $\mu=4$ is shown in Fig. 5, In this case the bending mode $m=2$ is developed initially in the inner disc regions $(r \lessgtr 2 L)$, see the saddle-like features in Fig. 6. Fig. 6) shows the distribution of $\zeta$ in the disc plane for certain moments between $t=0$ and $t=40$ (like in Fig. 5). The vertical heating and disc's flare due to the mode $m=2$ are very modest in this case. However, a non-linear one-arm asymmetric mode $m=1$ emerges after $t \gtrsim 3$ and the vertical heating gets more significant. The third stage of the heating starts at $t \gtrsim 10$ when 
the mode $m=0$ begins to dominate all over the disc. The growth rate of the vertical dispersion $c_{z}$ is especially high at this time: the disc scale height increases by a factor of $2-3$. The example considered shows the process of transformation between the bending modes and transition from the asymmetric mode $m=2$ to the axisymmetric one. In the case of a low-massive spherical subsystem of low mass, the favourable conditions for emerging the global bending instability get worse and the disc warp does not emerge in the disc for at least 20 rotation turns if the initial $\alpha_{z}=c_{z} / c_{r}$ is high enough.

If the initial template was too far from the stability limit, the disc "jumps over" the stability limit, and then its final state essentially exceeds this limit. Thus finally produces overstabilized and too thick disc. Since such stellar discs are not obviously observed, we have to assume that the initial state of the discs was not too far from the stability limit. Note that it agrees with a gradual building of stellar discs from gas or via minor merging when the initial discs do not emerge instantly but are built for a continuous time from small fragments, which adjust to the discs stability limit.

\subsection{Bending of bars}

In the course of experiments with low massive halo $(\mu \lesssim 1.5)$, if the initial state of disc is gravitationally unstable, a bar can emerge. The bar formation is accompanied by its warping (as reported by Raha et al. 1991).

Bending of the bar can be a result of global instability of the bar-mode during its initial stage when the bar forms in the initially thin cold disc. Fig. 7 shows the bar bendings that increase the vertical velocity dispersion. Amplitude of the bar warps decreases essentially when the bar thickness increases. We stress that once the bar has been formed, it stops the further possible developing of the global bending modes, and first of all it destroys the axisymmetric instability with mode $m=0$.

The bar formation runs faster while the initial disc is cold (i.e. for small values of Toomre's parameter $Q_{T}$ ) and as a result, amplitude of bar's bending mode increases (Fig. 7). If the initial disc lays in a slightly subcritical state $\left(c_{r}\right.$ is just below the level that provides the stability to the global bar-mode), the bar emerges very slowly, and it is stable against the bending perturbations, i.e. the bending of a bar does not necessarily emerge in the modelling.

\subsection{The ratio $c_{z} / c_{r}$}

The key parameter responsible for stability of the stellar discs is the ratio $\alpha_{z}=c_{z} / c_{r}$. To stabilize the global bending instabilities in the case of low values of relative mass of the spherical subsystem in a model, the value of $c_{z} / c_{r}$ has to be greater than $0.3-0.37$, as it was figured out from linear analysis of simple models (Poliachenko \& Shukhman 1977 Araki 1986, Merrit \& Sellwood 1994).
Let's consider a bulgeless model with a moderate halo $\mu=1$, see Fig. 1 and 2. The initial (curve 1) and final (curve 2) distributions of $\alpha_{z}(r)$ are shown in Fig. 8 8 . The vertical heating generated by the axisymmetric bending mode is as strong that the averaged over the disc ratio $\left\langle c_{z} / c_{r}\right\rangle=0.74$.

Once the relative mass of halo is chosen to be higher, the ratio $c_{z} / c_{r}$ needed to marginally stabilize the bending perturbations (we refer to it as the critical ratio $\alpha_{z}^{\text {crit }}$ hereafter) gets less. One can see the initial (curve 1) and final (curve 2) distributions of $\alpha_{z}(r)$ for the case of very massive halo with $\mu=4$ in Fig. $8 \mathrm{~b}$. The curve 3 shows the initial distribution of $\alpha_{z}(r)$, which is necessary to provide stability against the global bending modes. At the same time, at the disc periphery $0.27<c_{z} / c_{r}<0.37$.

A distinctive feature of the considered models at the threshold of bending stability is the non-uniformity of $c_{z} / c_{r}$ along the radius (see Fig. 8). For the case of moderate halo $(\mu \lesssim 1)$ with the initial distribution of $c_{r}(r)$ that suppresses the bar instability, the critical value of $\alpha_{z}^{c r i t}$ is a descending monotonous function of $r$. Its value ranges from $0.5 \div 0.6$ at the central regions to $0.3 \div 0.4$ at the periphery (see Fig. 3).

\section{Modelling of selected edge-on galaxies}

In order to compare our model predictions with observations, we choose eight spiral edge-on galaxies. Structural parameters for five of them: NGC 4738, UGC 6080, UGC 8286, UGC 9442 and UGC 9556, were taken from Bizyaev \& Kajsin 2004 Superthin edge-on galaxy UGC 7321 was studied by Matthews 1999, Matthews 2000, Radial distributions of the stellar velocity dispersion are available in addition to rotation curves and structural parameters for two large and nearby galaxies NGC 891 and NGC 5170 van der Kruit \& Searle 1981, Morrison et al. 1997, Bottema et al. 1991, Bottema et al. 1987). All the galaxies except NGC 891 and NGC 5170 indicate a negligible presence of bulge, which simplifies the modelling.

We evaluate the following free parameters of the model: $\mu=M_{s} / M_{d}$, the radial scale of the halo $a_{h}$ and the disc central surface density $\sigma_{0}$. We look for the optimal agreement between the calculated and observed disc thickness and rotation curve simultaneously. Here $M_{d}$ is the galactic disc mass; $M_{s}=M_{h}+M_{b}$ is the mass of the spherical component which comprises of the halo and bulge in general case. Note that since almost all our galaxies have no visible bulge, their spherical component in the most cases means the galactic dark halo.

We assume that the density distribution in stellar discs and bulges (if used) follows the brightness distribution. It corresponds to assumption of constant massto-light ratio.

The structural parameters of the galaxies are figured out for the Hubble constant $H_{0}=75 \mathrm{~km} \mathrm{~s}^{-1} \mathrm{Mpc}^{-1}$. The 
Table 1. Observational parameters of selected edge-on galaxies
$5.2 \cdot 10^{10} M_{\odot}, \sigma_{0}=1200 M_{\odot} / \mathrm{pc}^{2}$ and $V_{c, d} /\left.V_{c}\right|_{r=2.2 L}=$ 0.87 .

\begin{tabular}{|c|c|c|c|c|c|c|}
\hline Name & $\begin{array}{l}D \\
\mathrm{Mpc}\end{array}$ & $\begin{array}{l}L \\
\mathrm{kpc}\end{array}$ & $\begin{array}{l}\left\langle z_{0}\right\rangle \\
\mathrm{kpc}\end{array}$ & $\begin{array}{l}\left\langle h_{z}\right\rangle \\
\mathrm{kpc}\end{array}$ & $\begin{array}{r}R_{\max } \\
\mathrm{kpc}\end{array}$ & RC4.1.2. UGC 6080 \\
\hline NGC 891 & 9.5 & 4.9 & 0.98 & 0.49 & 21.0 & 1 The $V_{c}$ and $V^{o b s}$ in the left panels of Fig. 9 for \\
\hline NGC 4738 & 63.6 & 4.7 & 1.30 & 0.7 & 19.2 & 2 UGC 6080 are designated by the solid and dashed \\
\hline NGC 5170 & 20.0 & 6.8 & 0.82 & - & 26.2 & 3 curves, respectively. The model disc thickness is shown \\
\hline UGC 6080 & 32.3 & 2.9 & 0.69 & 0.48 & 9.9 & 4 in the right panel for $\mu=0.36$ (solid curve), $\mu=0.57$ \\
\hline UGC 7321 & 10.0 & 2.1 & $0.17^{b}$ & $0.14^{c}$ & 8.15 & 5 (dotted curve) and $\mu=0.9$ (dashed curve). The stellar \\
\hline UGC 8286 & 4.8 & 2.0 & 0.26 & 0.13 & 8.0 & 6 disc is thicker than it is observed for all models with \\
\hline UGC 9422 & 45.6 & 3.5 & 0.80 & 0.51 & 14.6 & ${ }^{4} \mu<0.6$. The best-fitting model gives the upper limit for \\
\hline UGC 9556 & 30.6 & $1.5(3.6)^{a}$ & 0.51 & - & 9.0 & $4_{\text {the disc mass }} M_{d}=5.3 \cdot 10^{10} M_{\odot}$ and $\sigma_{0}=1200 M_{\odot} / p c^{2}$ \\
\hline
\end{tabular}

latter one has larger scale length.

${ }^{b}$ the scale is given for the disc's periphery in $\operatorname{case} \operatorname{sech}\left(z / z_{c h}\right)$.

${ }^{c}$ the scale is given for the disc's centre.

Here $D$ is the distance to the galaxy, $L$ is the exponential disc scale length, $\left\langle z_{0}\right\rangle$ is the scale height for the $\operatorname{sech}^{2}$ shape of the vertical luminosity profile, $\left\langle h_{z}\right\rangle$ is the scale height for the case of exponential vertical profile, $R_{\max }$ is the disc cut-off radius. References to the rotation curves: 1 Sancisi 1979 Begeman et al. 1991, 2 - Giovanelli et al. 1997 3 - Bottema et al. 1987 4 - Karachentsev \& Zhou 1991, 5 Matthews 1999 6-Bottema et al. 1986

name of galaxy, adopted distance $D$, scale length $L$, observed mean scale heights $\left\langle z_{0}\right\rangle$ and $\left\langle h_{z}\right\rangle$ (corresponding to $\operatorname{sech}^{2}$ and exp luminosity distributions in the vertical direction, respectively), stellar disc radius $R_{\max }$, and reference to the source of the rotation curve are shown in Table 1

\subsection{Notes on individual objects}

Below we present some results and notices on modelling of the galaxies. All masses of galactic components given below are estimated within the limits of $R_{\max }$ from Table 1 .

\subsubsection{UGC 9422}

Fig. 9 shows the rotation curves $V^{\text {obs }}$ together with the model circular velocity curves $V_{c}$. $V^{o b s}$ are shown for $\mu=0.35$ and $\mu=0.6$ (dashed and dash-dotted curves, respectively). The "edge-on" values of $V_{c}$ are plotted for the same $\mu=0.35$ and $\mu=0.6$ (dotted and solid curves, respectively). The model radial distributions of $z_{0}$ are shown in the top right panel in Fig. 9. Model with low massive halo ( $\mu=0.4$, the solid curve) produces too thick stellar discs. It rules out the maximum disc model in which the central surface density $\sigma_{0}=1440 M_{\odot} / \mathrm{pc}^{2}$ and the disc contributes $94 \%$ into the circular velocity $\left(V_{c}^{d i s c} / V_{c}\right)$ at $r=2.2 \mathrm{~L}$. The acceptable agreement with the observed disc thickness can be achieved at $\mu \simeq 0.6$ (the dotted curve). Haloes with $\mu \gtrsim 1$ make the disc to be too thin (the model with $\mu=1.6$ is shown by the dashed curve in the corresponding panel of Fig. 9]. The bestfitting model has parameters: $M_{d}=8.6 \cdot 10^{10} M_{\odot}, M_{h}=$

\subsubsection{UGC 8286}

Left panel for UGC 8286 shows only $V^{o b s}$ for two models: $\mu=0.7$ (solid curve) and $\mu=1.6$ (dotted curve). Right panels show radial distribution of observed disc thickness for the same cases (solid and dotted curves, respectively). It is a good illustration that a rotation curve can be successfully explained with a wide range of $\mu$ whereas the disc thickness (right panel in Fig. 96 helps to choose the proper model. The best-fitting disc central surface density is the lowest in our sample that reveals the LSB nature of this galaxy.

\subsubsection{UGC 9556}

The radial photometric profile of UGC 9556 cannot be described by a single exponent. We find that It is better fit by two exponents with the scale lengths 1.5 and 3.6 $\mathrm{kpc}$ (Bizyaev \& Kajsin 2004). The notation in the left panel is kept the same as for UGC 6080. In the right panel, we show the model thickness for $\mu=0.9$ (dotted curve) and $\mu=1.9$ (solid curve). The best-fitting model with $\mu \approx 0.9$ produces a bar which can be responsible for the deviations from the exponential law in the inner regions of the model disc.

\subsubsection{UGC 7321}

Thin stellar disc of UGC 7321 and a lack of bulge indicates that the galaxy owns a massive dark halo. The notation in the left panel is kept the same as for UGC 6080. In the right panel we show the model thickness for $\mu=3.4$ (dotted curve) and $\mu=1.8$ (solid curve). The best-fitting model with $\mu=3.4$ has $M_{d}=0.53 \cdot 10^{10} M_{\odot}$ and $M_{h}=1.8 \cdot 10^{10} M_{\odot}$. The stellar disc contributes $\simeq 60 \%$ into the circular velocity at $r=2.2 \mathrm{~L}$. The central surface density in this case is only $\sigma_{0}=220 M_{\odot} / \mathrm{pc}^{2}$. This highlights the low surface brightness nature of the disc inferred by Matthews 1999. We investigate a case of the bar formation in this galaxy, according to the hypothesis by Pohlen et al. 2003 and make the conclusion that it cannot exist in UGC 7321, according to our modelling. 


\subsubsection{NGC 4738}

This galaxy has a bulge according to the photometric data, but its luminosity is less than $3 \%$ of the disc's that enables us to consider bulgeless models for NGC 4738. In the right panel we show the model thickness for $\mu=0.6$ (dotted curve) and $\mu=0.3$ (solid curve). The halo with intermediate mass $\mu \gtrsim 0.5$ is suitable for this galaxy. The models with less massive halo $(\mu=0.3)$ produce too thick discs for this galaxy.

\subsubsection{NGC 5170}

An extended bulge is required to match observed RC for this galaxy. On the other hand, the photometry indicates a presence of very small bulge in this galaxy. The observed shape of $\mathrm{RC}$ with rather low resolution so a disagreement with the model $\mathrm{RCs}$ is not unexpected at $r \lesssim 2 \mathrm{kpc}$. Fig. 10 shows the RC and radial distributions of velocity dispersions calculated for the maximum disc model and for the model with $\mu=1.6$. The latter case provides a better agreement with observational data for both RC and velocity dispersion. Moreover, the maximum disc model requires the main disc thickness to be $1.1 \mathrm{kpc}$ whereas the observations suggest $0.82 \mathrm{kpc}$. The model with $\mu=1.6$ produces the projected mean scale height $0.8 \mathrm{kpc}$. In general, the agreement between RC and both velocity dispersion (Fig. 10k) and stellar disc thickness is matched when $\mu \gtrsim 1.5$.

\subsubsection{NGC 891}

This galaxy has a budge (Bottema et al. 1991 Morrison et al. 1997), and we took it into account evaluating our numerical models. Published values of the vertical exponential scale height for old stellar disc varies from $400 \mathrm{pc}$ to $650 \mathrm{pc}$ (van der Kruit \& Searle 1981 Bahcall \& Kylafis 1985. $\quad$ Shaw \& Gilmore 1989. Morrison et al. 1997, Xilouris et al. 1999). We assume the set of structural parameters inferred for NGC 891 by van der Kruit \& Searle 1981 .

The observed and model radial distributions of the stellar velocity dispersion (Bottema et al. 1991), stellar disc thickness and rotation curves are shown in Fig. 11 The stellar velocity dispersion in the centre of the galaxy reaches $160 \mathrm{~km} / \mathrm{s}$ (Bottema et al. 1991) for stars in the bulge, and the radial component of the disc velocity dispersion is $c_{r} \leq 120 \mathrm{~km} / \mathrm{s}$. Existence of a molecular ring in the galaxy at $r \simeq 3.5 \mathrm{kpc}$ enables us to assume that the galaxy harbours a bar (Sofue \& Nakai 1992).

Once the radial distribution of the stellar velocity dispersion is taken into account together with condition of marginally stable stellar disc, successful model should have $\mu>1.5$. Note that the use of an alternative $\mathrm{RC}$ from Khoperskov et al. 2001 gives $\mu=1.8$ with other similar parameters.

Fig. 11b shows the radial distribution of the disc exponential vertical scale height for the set of $M_{h} / M_{d}=$ $0.4,0.7$ and 1.7. The stellar disc thickness indicates that the dark and luminous masses are roughly equal to each other within $R_{\max }$. The value of $\mu$ inferred from the disc thickness is slightly less than that estimated from the velocity dispersion probably due to additional factors that increase the disc thickness in addition to the bending instability.

The disc mass of $1.5 \cdot 10^{11} M_{\odot}$ estimated with the shape of rotation curve (Bottema et al. 1991) corresponds to a very high value of mass-to-light ratio 13.5 \pm 4 . Our method yields $5 \div 10$ for this ratio. This introduces an argument against the the maximum disc in NGC 891.

Finally, we consider a case of two stellar discs of different thickness in the galaxy. This assumption was made by Morrison et al. 1997. We found that such an assumption almost does not change the final best-fitting value of $\mu$.

\subsection{Relative thickness of stellar disc versus relative mass of spherical subsystem}

A tight connection between the stellar disc thickness $z_{0} / L$ and relative mass of spherical subsystem follows from our modelling, see Fig. 12. Each circle in the Fig. 12 corresponds to a model galaxy with different parameters of the disc and spherical subsystem. The figure includes our modelling of the objects studied by Mikhailova et al. 2001, Zasov et al. 1991 in addition to results of the present paper. The upper curve corresponds to mostly bulgeless models. The lower one designates the loci of models with noticeable and compact bulges. As it can be seen in Fig. 12, the presence of a bulge introduces a little scatter to the $<z_{0}>/ L \div M_{s} / M_{d}$ dependence that can be easily parameterized in dependence of the bulge mass. This relation can be used to estimate $M_{s} / M_{d}$ if $z_{0} / L$ is known from observations.

\subsection{Results and discussion}

The parameters of best-fitting models for our galaxies are summarized in Table 2 . In addition to the evaluated model parameters, we estimate the dark-to-luminous mass ratio $M_{\text {dark }} / M_{\text {lum }}$ (it equals to $\mu$ if no bulge was assumed in the model. In the case of bulge we derive the ratio $M_{\text {dark }} / M_{\text {lum }}$ as $M_{h} /\left(M_{d}+M_{b}\right)$, where $M_{b}$ denotes mass of the bulge. As one can see from the table, the fractions of dark and luminous matter in more than a half objects from our sample are roughly the same. The exceptions are the thin galaxies NGC 5170, UGC 7321 and UGC 8286 which have massive dark haloes and low surface brightness discs (low $\sigma_{0}$ in our modelling). The same may be referred to the external low surface brightness disc of UGC 9556.

In Fig. 13 we compare the relative disc thickness $\left(z_{0} / L\right)$ and its central surface density (here we consider only thin external LSB disc in UGC 9556). The figure shows a good correlation between the disc relative thickness and its central surface density. Our 
sample is small but much more data from photometry can be incorporated. Thus, the disc central surface brightness was compared with their thickness by Bizyaev \& Mitronova 2002, Bizyaev \& Kajsin 2004 Bizyaev \& Mitronova 2009. A good observational correlation was found: the thicker the disc, the greater its central surface brightness. The latter parameter reveals the central surface density of disc and corresponds to $\sigma_{0}$ in Fig. 13. It means that the correlation in Fig. 13 comes from two independent methods: structural studies and numerical modelling.

The relative thickness of the disc is a crucial parameter in our modelling, and is relevant to the relative mass of the spherical component. The relative thickness may span quite a wide range from 3 to 12 (see van der Kruit \& Searle 1982, de Grijs 1997. $\quad$ de Grijs \& van der Kruit 1996 Bizyaev \& Mitronova 2002, Bizyaev \& Kajsin 2004 Bizyaev \& Mitronova 2009). According to our conclusions it means that the relative mass of the spherical subsystem varies significantly from galaxy to galaxy.

All these values are dimensionless whereas the central surface density of stellar disc depends on the amplitude of rotation curve which comes from observations. Hence, our model could reproduce a rather thick low surface brightness disc (say, without a spherical subsystem at all) or, in a contrary, a very dense stellar disc surrounded by massive halo which makes it to be very thin. As it can be seen from Fig. 13 and Bizyaev \& Mitronova 2002, such kind of galaxies were not found among both normal and low surface brightness spirals. It suggests that some factors beyond our model assumptions regulate the connection between the disc surface density and dark halo masses. Thus can be a result of evolution as well as a consequence of darkluminous matter interaction on the stages of galaxies formation.

Additional factors as density waves, tidal perturbations or GMO can also be developed in stellar discs and amplify the vertical motions in disc. Nevertheless, developing the bending perturbations is the most powerful mechanism heating up the discs and our model results should not underestimate $\mu$ very much. As is was shown by Zasov et al. 2004, our approach works well for the stellar discs of spiral galaxies Sa-Sd.

Structural parameters of considered galaxies were obtained for mostly old stellar population (Bizyaev \& Kajsin 2004), therefore we do not expect large variations of mass-to-light ratio in the vertical direction.

The galactic dust layer in edge-on galaxies creates a problem of reliability of structural parameters. Nevertheless, the parameters for our galaxies were obtained using off-plane regions (see full discussion in Bizyaev \& Kajsin 2004 and Bizyaev \& Mitronova 2002). It should significantly decrease the influence of dust to results of our modelling.
Realistic simulations should include "live" halo rather that that approximated by a fixed potential. For the case of the "live" halo our N-body simulations illustrate an extreme case and the ratio $M_{s} / M_{d}$ found in our models shows the lower limit for the halo-to-disc mass ratio. We found an ability to run our model upgraded with a "live" halo for one of our galaxies, UGC 8286 . Figure 14 is the same as the third upper and right panel in Figure 9(i.e. its part that concerns the disc thickness in UGC 8286) updated with more realistic N-body simulations. The asterisks (upper curve), diamonds (next lower), and triangles designate the models with $\mathrm{N}_{\text {halo }}$ $=2 \cdot 10^{5}, 4 \cdot 10^{5}$ and $6 \cdot 10^{5}$, respectively, where $\mathrm{N}_{\text {halo }}$ is the number of bodies in the dark halo in our N-body simulations. Note that the curves for $\mathrm{N}_{\text {halo }}=4 \cdot 10^{5}$ and $6 \cdot 10^{5}$ are almost identical. As we can see, the more realistic case of the three considered haloes suggests a more massive spherical component than that for the fixed potential, but the difference is of the order of $10 \%$. Thus, we encourage to consider our halo-to-disc mass ratio given in Table 2 as a lower limit for the real value of $M_{\text {dark }} / M_{\text {lum }}$ that is nevertheless not far away from it.

\section{Conclusions}

1. Development of the bending instabilities in stellar galactic discs is studied with the help of N-body numerical simulations. The axisymmetric bending mode ( $m=0)$ is found to be the strongest factor which may heat up the disc in the $z$-direction. If no bar is developed, the bending modes $m=1$ and $m=2$ are not significant contributors to the process of the disc thickening. The most significant growth of the disc thickness occurs at the initial non-linear stage of the bending formation. Once the bending was destroyed, the vertical heating gets less effective. The lifetime of the bending mode $m=0$ is higher for the larger relative mass of the spherical subsystem $\mu$. We show that initially very thin discs increase their thickness much more rapidly than those started from a marginally subcritical state. As a result of the global bending instability development, the final values of the vertical velocity dispersion $c_{z}(r)$ and vertical scale height $z_{0}(r)$ can be larger than those required for the fulfillment of the disc stability condition. 2 . The critical value of the ratio $\alpha_{z}^{c r i t}=c_{z} / c_{r}$ is a function of the spherical subsystem parameters. At the threshold of stability, the value of $\alpha_{z}^{c r i t}(r)$ decreases with the distance to the centre. The value of $\alpha_{z}^{\text {crit }}$ can be twice as less at the periphery in the comparison with $\alpha_{z}^{\text {crit }}$ at the centre. The radial trend of $\left(c_{z} / c_{r}\right)^{\text {crit }}$ can be approximated as $c_{z} / c_{r} \propto \exp \left(-r / L_{\alpha}\right)$ with very long scale length $L_{\alpha} \simeq(5-6) \cdot L$.

3. The average relative vertical disc scale height $\left\langle z_{0}\right\rangle / L$ decreases when the relative mass of the halo $\mu$ increases. This can be utilized to estimate the mass of the spherical subsystem for edge-on galaxies.

4. In the frames of our approach, we conduct N-body modelling of eight edge-on galaxies and compare re- 
Table 2. Inferred parameters of the spherical and disc components in the considered edge-on galaxies

\begin{tabular}{lcccccccc}
\hline Name & $\begin{array}{c}a_{h} \\
\mathrm{kpc}\end{array}$ & $\begin{array}{c}M_{h} \\
10^{10} M_{\odot}\end{array}$ & $\begin{array}{c}M_{d} \\
10^{10} M_{\odot}\end{array}$ & $\begin{array}{c}\sigma_{0}^{\text {disc }} \\
\frac{M_{\odot}}{p c^{2}}\end{array}$ & $\begin{array}{c}a_{b} \\
\mathrm{kpc}\end{array}$ & $\begin{array}{c}M_{b} \\
10^{10} M_{\odot}\end{array}$ & $\mu$ & $M_{\text {dark }} / M_{\text {lum }}$ \\
\hline NGC 891 & 5.4 & 7.13 & 10 & 715 & 1.09 & 1.86 & $0.9(1.7)$ & $0.7(1.2)$ \\
NGC 4738 & 10.4 & 5.5 & 11 & 870 & - & - & 0.5 & 0.5 \\
NGC 5170 & 8.67 & 19.2 & 12.8 & 491 & 2.43 & 1.15 & 1.6 & 1.4 \\
UGC 6080 & 3.53 & 3.01 & 5.3 & 1230 & - & - & 0.6 & 0.6 \\
UGC 7321 & 2.16 & 1.8 & 0.53 & 220 & - & - & 3.4 & 3.4 \\
UGC 8286 & 3.1 & 0.76 & 0.47 & 203 & - & - & 1.6 & 1.6 \\
UGC 9422 & 3.8 & 5.2 & 8.6 & 1200 & - & - & 0.6 & 0.6 \\
UGC 9556 & 3.7 & 1.29 & $0.57(0.81)$ & $715(139)$ & - & - & 1.1 & 1.1 \\
\hline
\end{tabular}

Name of galaxy, scale length of dark halo, mass of halo, mass of disc, disc central surface density, scale length of bulge (if a bulge was included), mass of bulge, spherical-to-disc mass ratio $\mu$, and dark-to-luminous mass ratio.

The numbers in parentheses for UGC 9556 correspond to its outer disc. For NGC 891, the estimates of $\mu$ and $M_{\text {dark }} / M_{\text {lum }}$ that were obtained from the observed radial velocity dispersion are given in parentheses.

sults with published rotation curves and surface photometry data. For the cases of NGC 5170 and NGC 891 we additionally incorporate published data on the stellar radial velocity dispersion. The relative mass of spherical subsystem (the pure dark halo in most cases) is estimated for all the galaxies. We conclude that the mass of the dark halo within the optical limits in a half of our galaxies is of the order of their disc's mass. The exceptions are a thin LSB galaxy UGC 7321 whose the dark halo encompasses about $2 / 3$ of the total overall mass, and two more galaxies NGC 5170 and UGC 8286 with thin discs.

5. The central surface density of stellar discs in the galaxies of our sample correlates well with the relative thickness of the discs. This correlation may be utilized to estimate the thickness of stellar discs in non edge-on galaxies.

\section{Acknowledgments}

Authors wish to thank prof. A.V. Zasov for fruitful discussions and the anonymous referee for valuable comments. The research was partly supported by grants RFBR 09-02-97021 and by the Federal Target Program "Scientific and scientific-pedagogical personnel of the innovative Russia" 2009-21(7). The computations were partly conducted at the supercomputer SKIF MGU Chebyshev (Moscow State University).

\section{References}

van Albada, T., Bahcall, J., Begeman, K., Sancisi, R., 1985, ApJ, 295, 305

Araki, S., 1986, PhD thesis, Massachusetts Institute of Technology

Araki, S., Tremaine, S., 1986, Icar, 65, 83

Athanasoula, E., Sellwood, J.A., 1986, MNRAS, 221, 213

Bahcall, J., 1984, ApJ, 276, 156

Bahcall, J.N., Kylafis, N.D., 1985, ApJ, 288, 252

Bailin, J., Steinmetz, M., 2003, Ap.\& Space Sci., 284, 701

Binney J., Jiang, I.-G., Dutta, S., 1998, MNRAS, 297, 1237

Bizyaev, D., Kajsin, S., 2004, ApJ, 613, 886
Bizyaev, D., Mitronova, S., 2002, A\&A, 389, 795

Bizyaev, D., Mitronova, S., 2009, ApJ, 702, 1567

Bottema, R., Gerritsen, J., 1997, MNRAS, 290, 585

Bottema, R., Shostak, G., van der Kruit, P., 1986, A\&A, 167, 34

Bottema, R., van der Kruit, P., Freeman, K., 1987, A\&A, 178, 77

Bottema, R., van der Kruit, P.C., Valenijn, E.A., 1991, A\&A, 247, 357

Bottema, R., 1993, A\&A, 275, 16

Begeman, K.G., Broels, A.H., Sanders, R.H., 1991, MNRAS, 249, 523

Carlberg R.G., Sellwood, J.A., 1985, ApJ, 292, 79

Combes, F., 2002, New Astron. Reviews, 2002, 46, 755

Fuchs, B., von Linden, S., 1998, MNRAS, 294, 513

Fux, R., 1997, A\&A, 327, 983

Giovanelli, R., Avera, E., Karachentsev, I.D., 1997, AJ, 114, 122

Goad, J., Roberts, M., 1981, ApJ, 250, 79

Griv, E., Chiueh, T., 1998, ApJ, 503, 186

Griv, E., Yuan, C., Gedalin, M., 2002, ApJ, 2002, 580, 27L

de Grijs, R., 1997, PhD thesis, Univ. Groningen, The Netherlands

de Grijs, R., van der Kruit, P., 1996, A\&A Suppl., 117, 19

Hernquist, L., Heyl, J., Spergel, D., 1993, ApJL, 416, 9

Holley-Bockelmann, J., Mihos, J., 2001, AAS, 198.0815

Hunter, C., Toomre, A., 1969, ApJ, 155, 747

Iodice, E., Arnaboldi, M., Bournaud, F., Combes, F., et al., 2003, ApJ, 585, 730

Jenkins, A., Binney, J., 1990, MNRAS, 245, 305

Karachentsev, I., Zhou, S., 1991, Astron. Letters, 17, 135

Khoperskov, A., Zasov, A., Tyurina, N., 2001, Astron. Reports, 45,180

Khoperskov, A., Tyurina, N., 2003, Astron. Reports, 47, 443

Khoperskov, A., Zasov, A., Tyurina, N., 2003, Astron. Reports, 47,357

Khoperskov, A.V., Just, A., Korchagin, V.I. et al. 2007, A\&A, 473, 31

van der Kruit, P., Searle, L., 1981, A\&A, 95, 116

van der Kruit, P., Searle, L., 1982, A\&A, 110, 61

Mayer, L., Governato, F., Colpi, M., Moore, B., et al., 2001, ApJ, 559, 754

Matthews, L.D., Gallagher, J.S., van Driel, W., 1999, AJ, 118, 2751

Matthews, L.D., 2000, AJ, 120, 1764 
Mikhailova, E., Khoperskov, A., Sharpak, S. 2001, The Zstructure of Disk Galaxies. Numerical Experiments, in Stellar dynamics: from classic to modern, ed. L.P. Ossipkov, I.I. Nikiforov, (Saint Petersburg), 147

Mitronova, S., Bizyaev, D., AAS 207, 188.07, astro-ph/0602223

Morrison, H., Miller, E., Harding, P., Stinebring, D., Boroson, T., 1997, AJ, 113, 2061

Merritt, D., Sellwood, J., 1994, ApJ, 425, 551

Nilson, P., 1973, Uppsala General Catalogue of Galaxies, Uppsala Astr. Obs. Annaler, Band 6

Ostriker, J., Peebles, P., 1973, ApJ, 186, 467

Patsis P.A., Athanassoula E., Grosbol P., Skokos, Ch., 2002, MNRAS, 335, 1049

Pohlen, M., Dettmar, R.-J., Ltticke, R., Aronica, G., 2002, A\&A, 392, 807

Pohlen, M., Dettmar, R.-J., Ltticke, R., 2003, A\&A, 409, 485

Poliachenko, V.L., Shukhman, I.G., 1977, Soviet Astron. Lett., 3, 134

Polyachenko, V.L., Shukhman, I.G., 1979, Astronomy Reports, 23, 407

Raha, N., Sellwood, J., James, R., Kahn, F., 1991, Nature, 352, 411

Reshetnikov, V., Combes, F., 2002, A\&A, 382, 513

Reshetnikov, V., Sotnikova, N., 2000, Astron. Letters, 26, 277

Sancisi, R., Allen, R.J., 1979, A\&A, 74, 73

Sellwood, J., 1996, ApJ, 473, 733

Sellwood, J.A., Merritt, D., 1994, ApJ, 425, 530

Spitzer, L., Schwarzschild, M., 1951, ApJ, 114, 385

Spitzer, L., Schwarzschild M., 1953, ApJ, 118, 106

Shaw, M.A., Gilmore, G., 1989, MNRAS, 237, 903

Sofue, Y., Nakai, N., 1993, PASJ, 45, 139

Sotnikova, N., Rodionov, S., 2003, Asron. Letters, 29, 321

Sotnikova, N., Rodionov, S., 2005, Asron. Letters, 31, 15

Sotnikova, N., Rodionov, S., 2006, Asron. Letters, 32, 649

Toomre, A., 1966, Geophys. Fluid Dyn., No. 66-46, 111

Xilouris, E.M., Byun, Y.I., Kylafis, N.D., Paleologou, E.V., Papamastorakis, J., 1999, A\&A, 344, 868

Valluri M., 1994, ApJ, 430, 101

Vandervoort, P., 1991, ApJ, 377, 49

Velazquez, H., White, S., 1999, MNRAS, 304, 254

Weinberg, M., 1998, MNRAS, 299, 499

Whitmore, B., McElroy, D., Schweizer, F., 1987, ApJ, 314, 439

Zasov, A., Makarov, D., Mikhajlova, E., 1991, Astron. Letters, 17,374

Zasov, A.V., Khoperskov, A.V., 2003, Astron. Letters, 29, 437

Zasov, A., Khoperskov, A., Tiurina, N., 2004, Astron. Letters, 30,593 

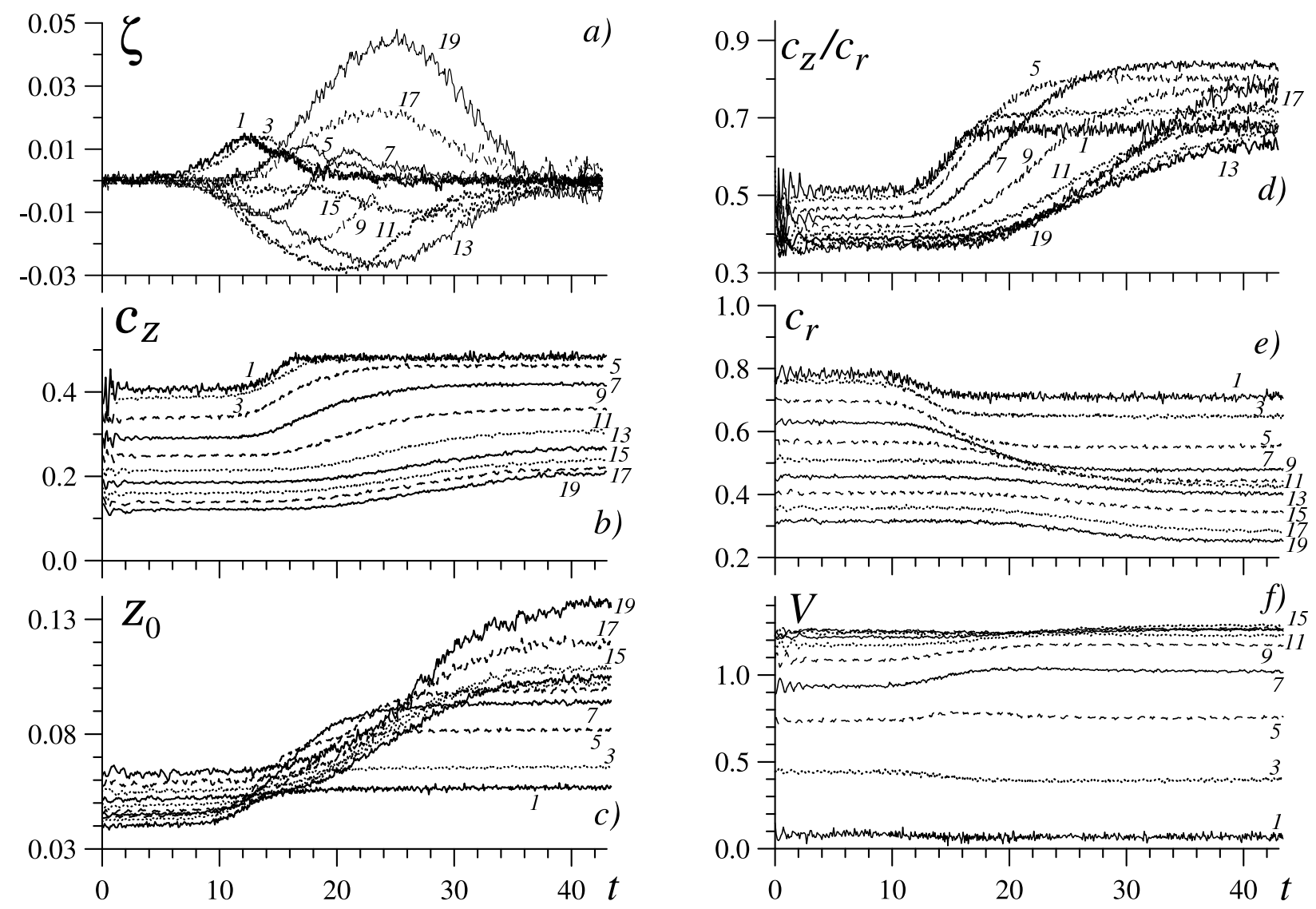

Fig. 1. Evolution of the vertical structure parameters of a model stellar disc for the case of $\mu=1, a=L$ when the axisymmetric bending mode is being developed: a) the vertical coordinate of the disc's local centre of mass $\zeta$, b) vertical component of the velocity dispersion $c_{z}$, c) disc scale height $z_{0}$, d) vertical-to-radial velocity dispersions ratio $c_{z} / c_{r}$, e) $c_{r}$, f) rotational velocity of particles in the disc. Different curves are drawn for the following set of distances to the centre: $r_{j}=4 L \cdot(0.05 j-0.025)$, where $j$ is shown by the corresponding curve. All the parameters are averaged along the azimuthal coordinate. 

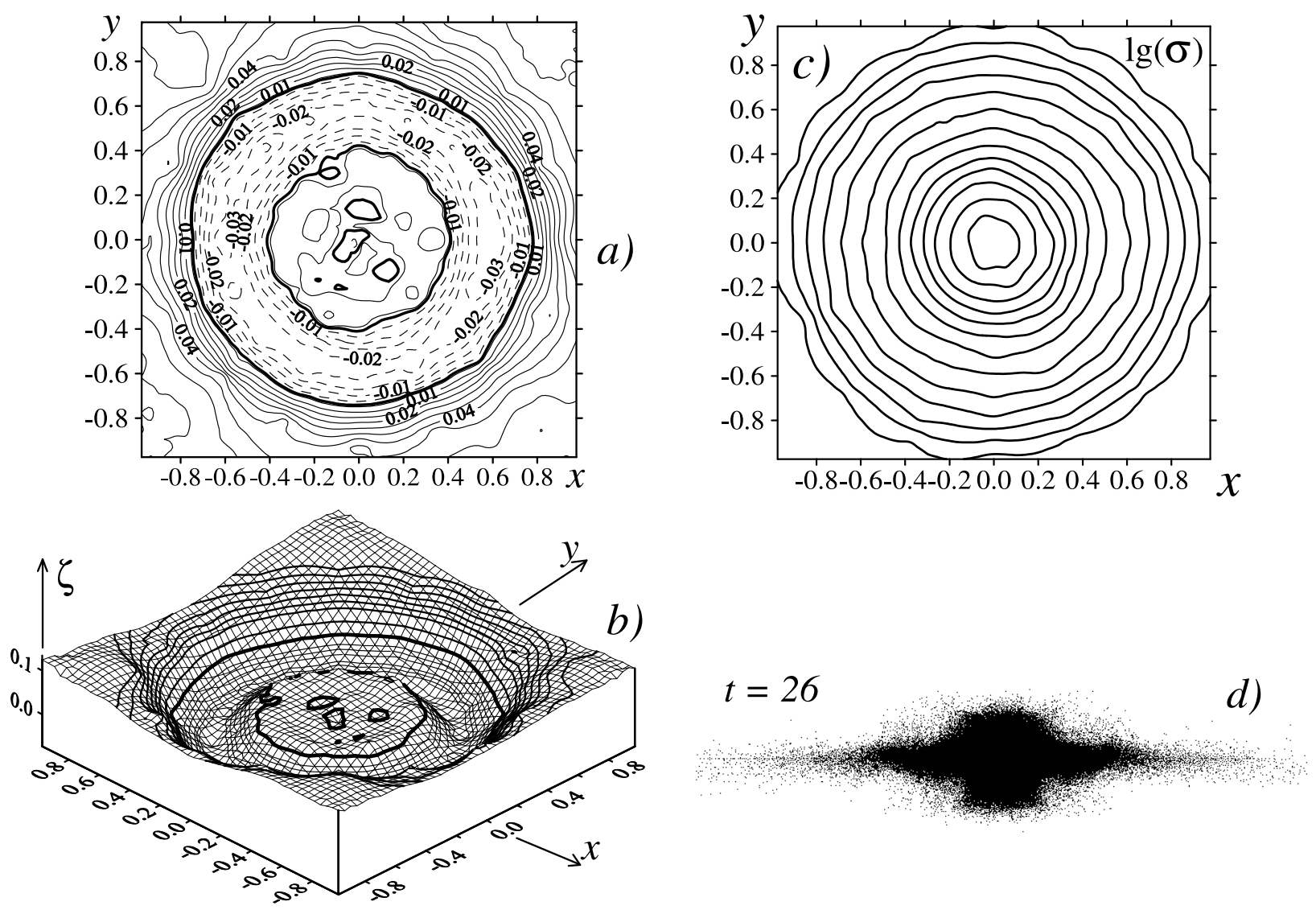

Fig. 2. The shape of a model disc that experiences a development of the bending instability at the moment $t=25.4$ for the model shown in Fig. 1. The isolines denote the distribution $\zeta(r, \varphi)$ in the plane of the disc (a) and in the $\mathrm{x}-\mathrm{y}$ plane $\zeta(x, y)(\mathrm{b})$. The bold solid line represents $\zeta=0$. c) Isolines of logarithm of the surface density are shown at the same moments as in the panel (a). The axial symmetry in the surface density distribution can be seen in the figure. d) Edge-on view at the disc on the stage of the global axisymmetric mode development. The vertical/radial aspect ratio in Figure is increased by the factor of 4 . 

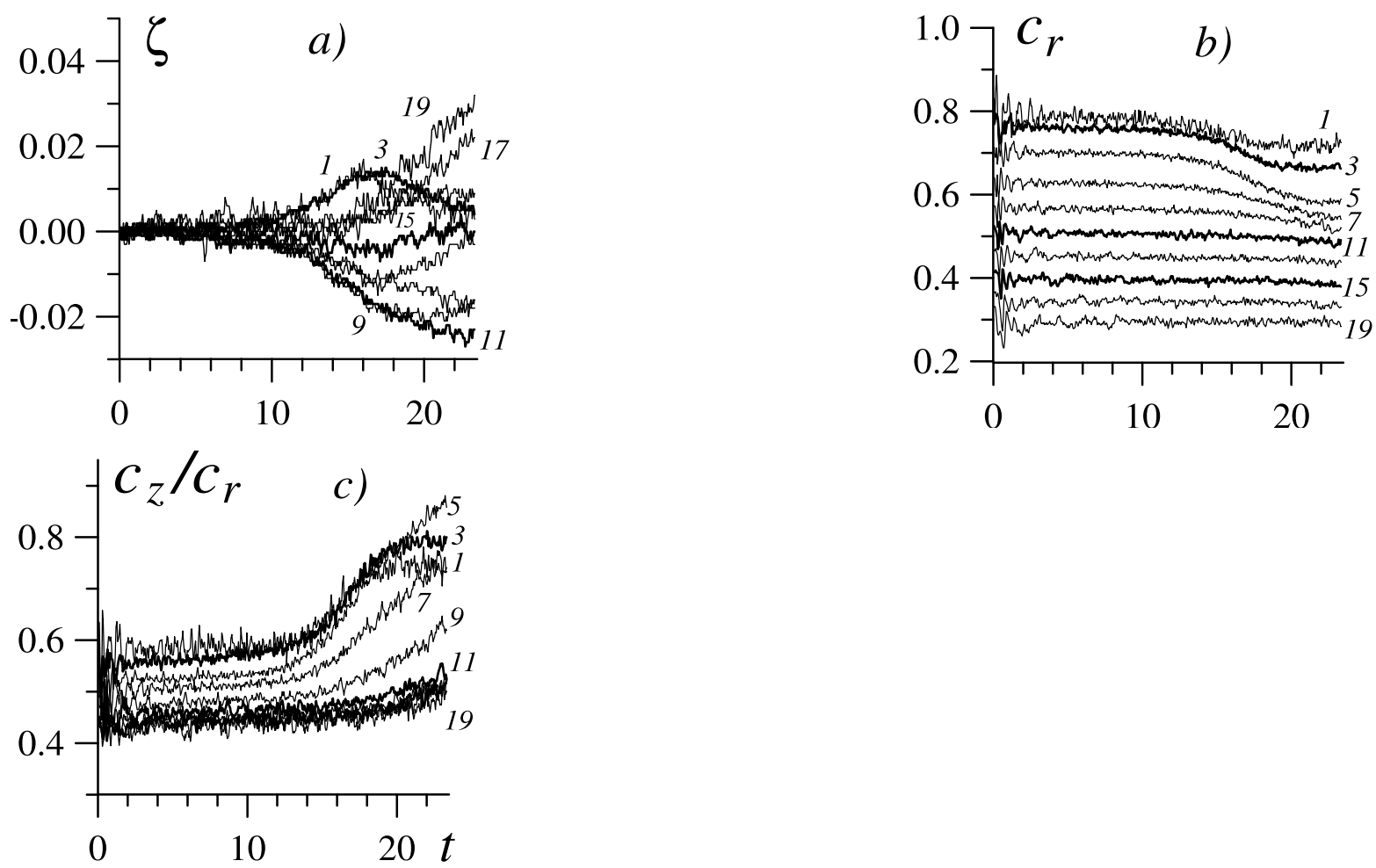

Fig. 3. Results of the same model as in Fig. 1 but evaluated with the help of PP code (see text). 

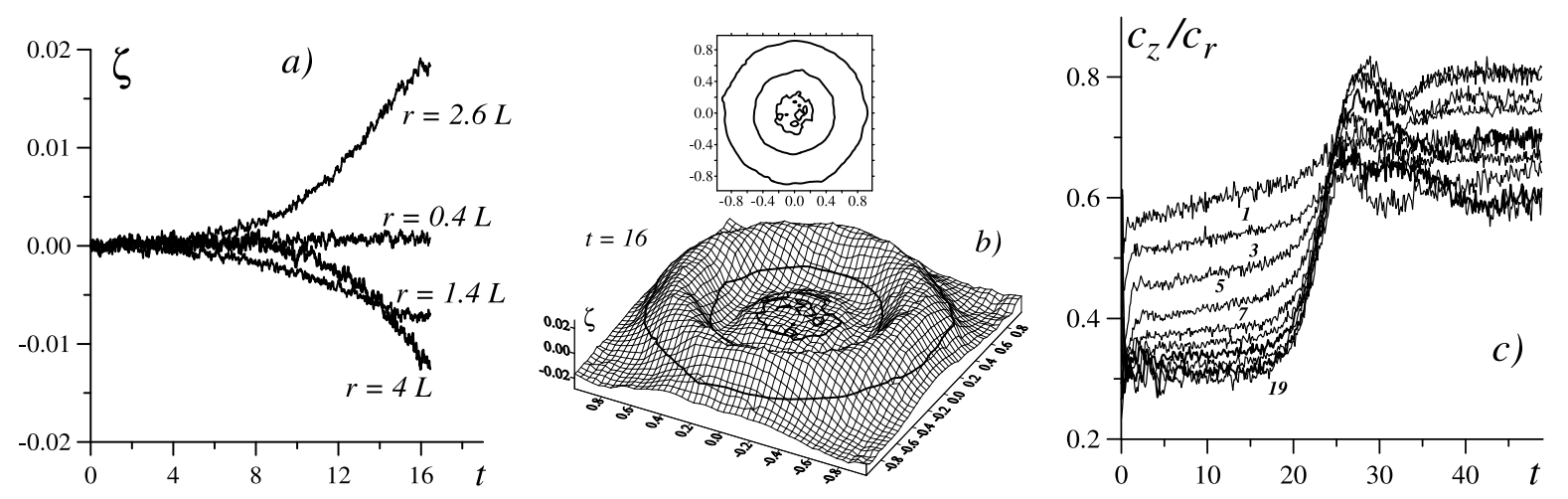

Fig. 4. Initial conditions of this model with $\mu=4$ provide the central parts of disc with stability, and the bending mode $m=0$ develops itself in the outer regions $(r>L)$. a) Initial stages of evolution of $\zeta$ at different distances from the centre. b) Distribution of $\zeta=0$ through the disc. The thick line denotes the isoline $\zeta=0$. c) The heating of a model disc without a halo at all, in presence of a bulge $\left(M_{b}=0.25 M_{d}, b=0.2 L\right)$, when the mode $m=0$ is being developed. The ratio $c_{z} / c_{r}$ does not rise significantly in regions of the bulge. The notation is kept the same as in Fig. 1. 


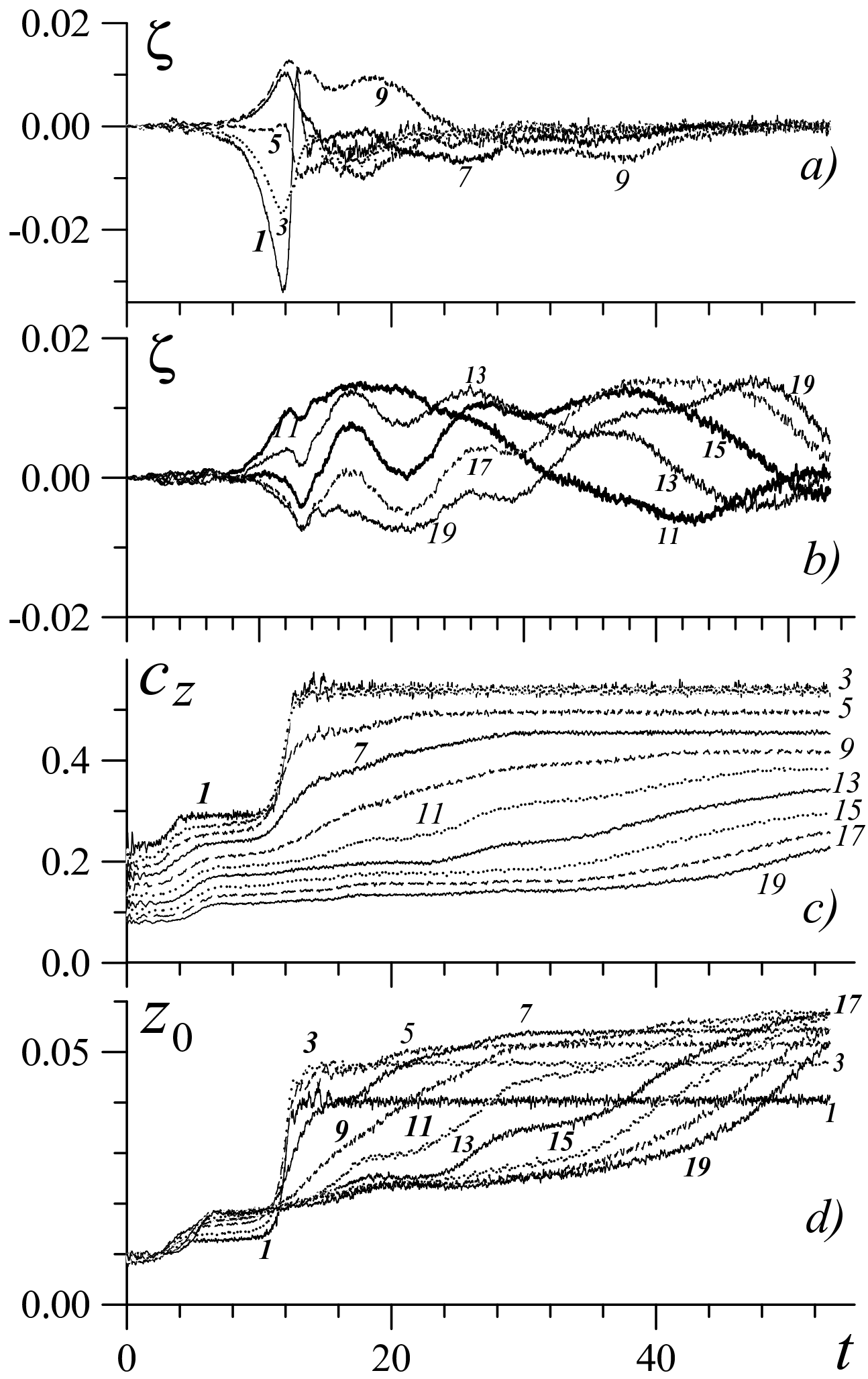

Fig. 5. The same as in Fig. 1 but for the model with $\mu=4$. Distributions of $\zeta$ for the internal and external regions are shown in separated two top panels. 

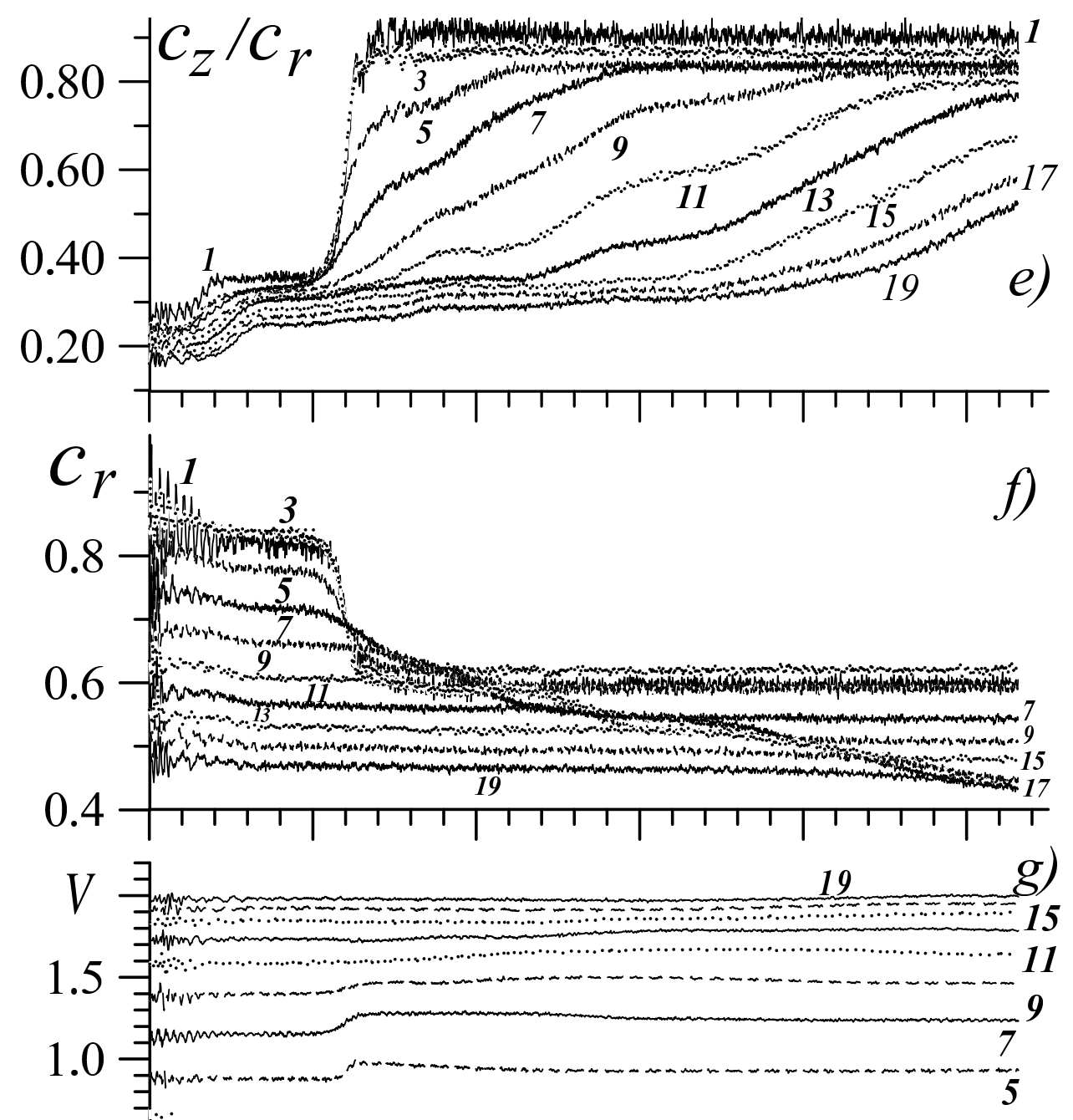

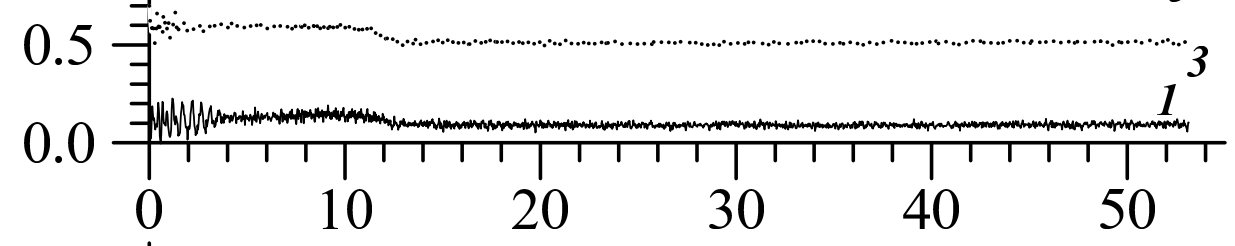

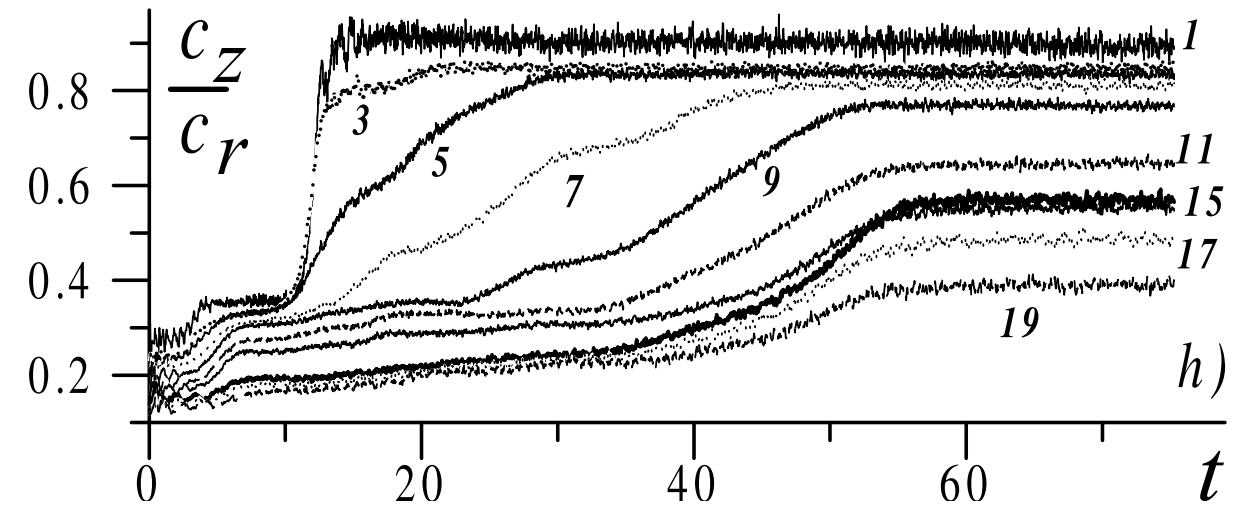



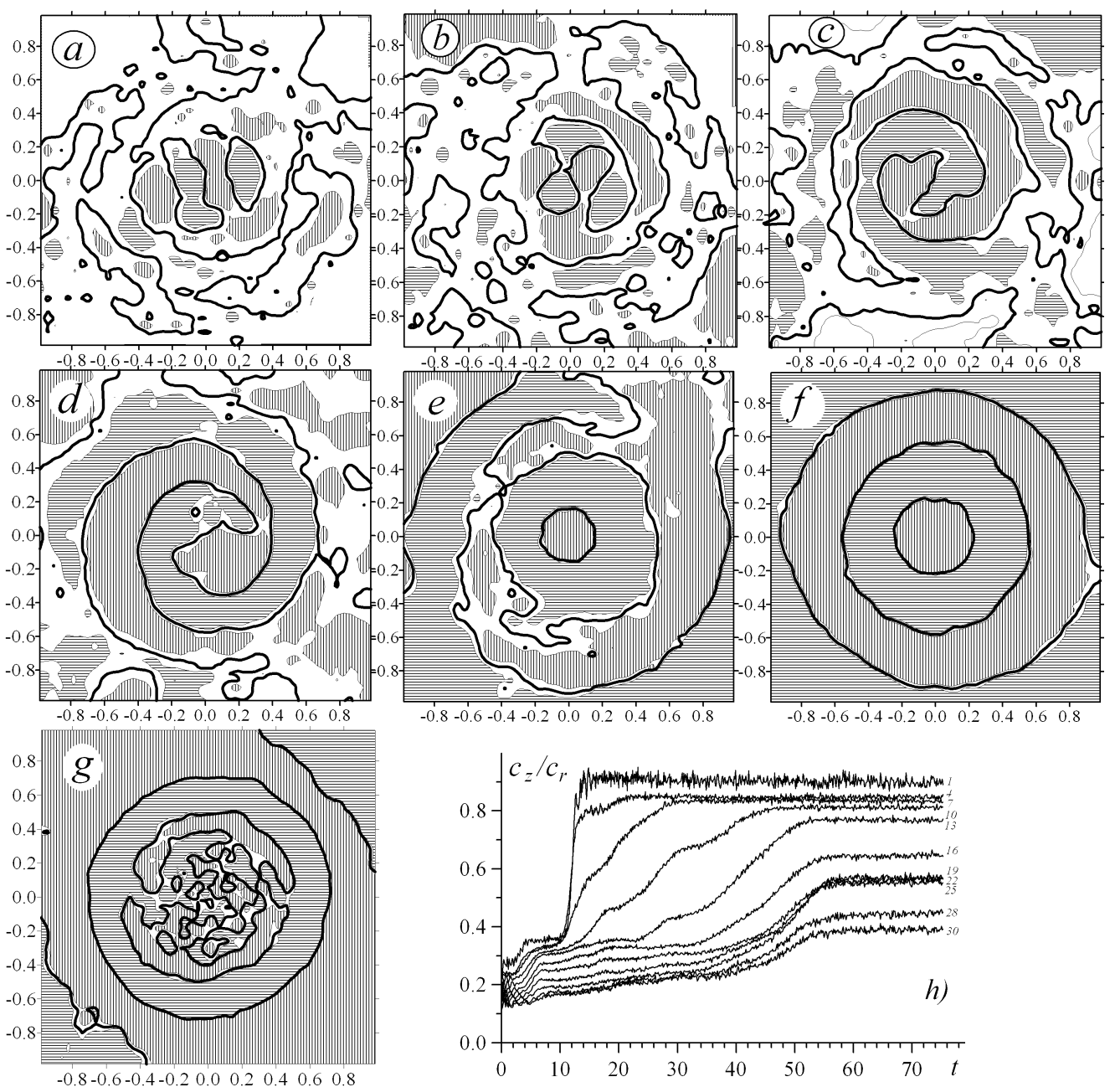

Fig. 6. Distribution of $\zeta(x, y)$ in the disc plane at different moments of evolution: a) $t=2.7$ - the mode $m=2$ is developing in the central region, b) $t=3.1$ $-m=2 \rightarrow m=1$ reorganization, c) $t=3.6$, d) $t=4.2$ - the well-developed one-arm mode $m=1$ is seen, e) $t=10.1-m=1 \rightarrow m=0$ reorganization, d) $t=16.4$ - the well-developed one-arm mode $m=0$ emerges, e) $t=51-$ all perturbations in the centre $r<L=0.25$ have almost disappeared. The axisymmetric perturbations can be seen in the intermediate regions whereas the mode $m=2$ dominates at the periphery (f, g). h) The time dependence of $c_{z} / c_{r}$ at different radii. 

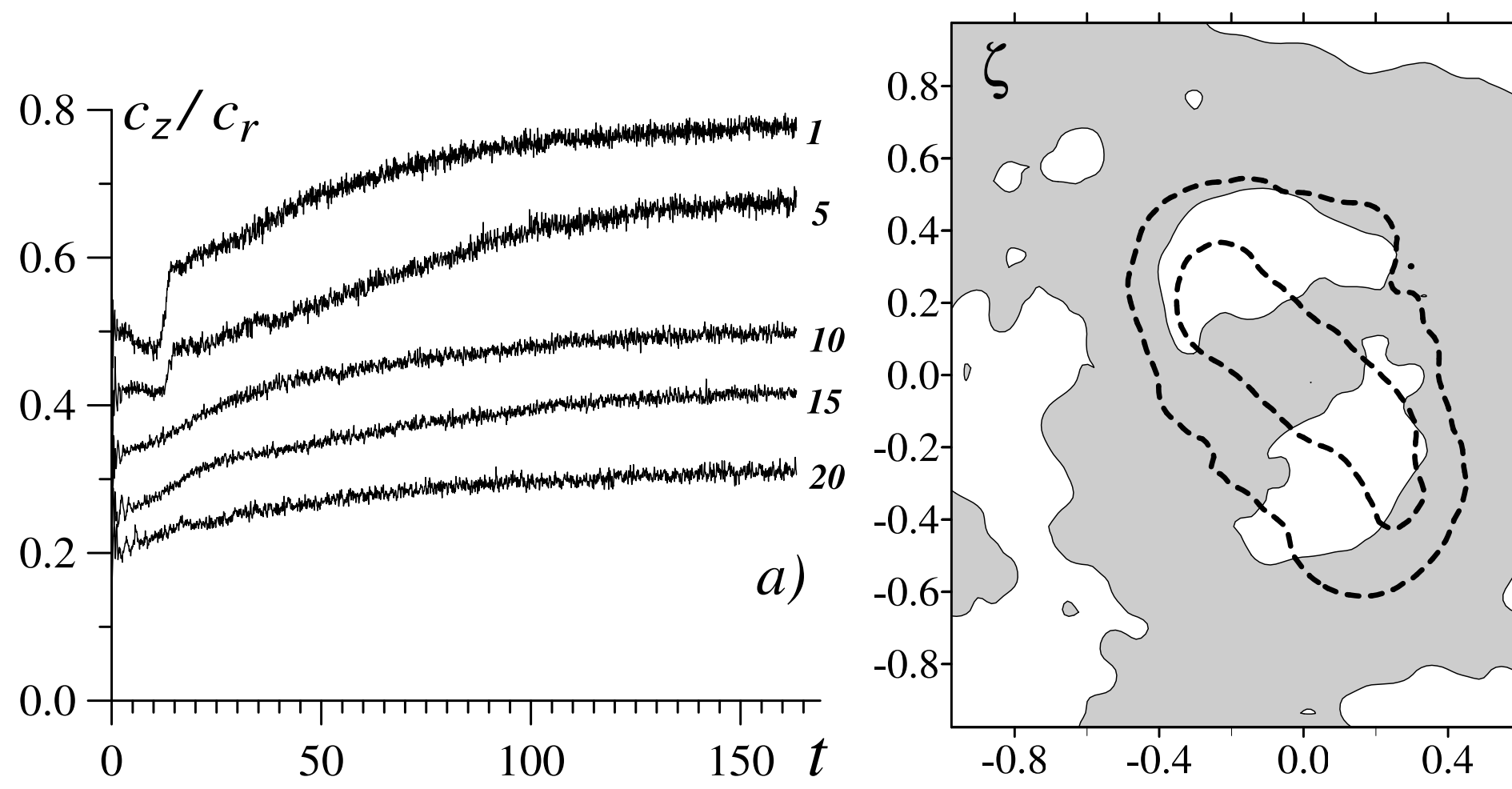

Fig. 7. Formation of bar. a) Evolution of the ratio $c_{z} / c_{r}$ at different radii in the model with bar. b) Distribution of $\zeta$ in the plane $x-y$ reveals structure of the bending mode at $t=15$. The thin solid line designates $\zeta=0$. The dotted lines show isodenses of the bar. Warping of the bar is the reason for rapid vertical heating in the central region (lines 1 and 5 at the range of $t=10 \div 20$ ), see text. 

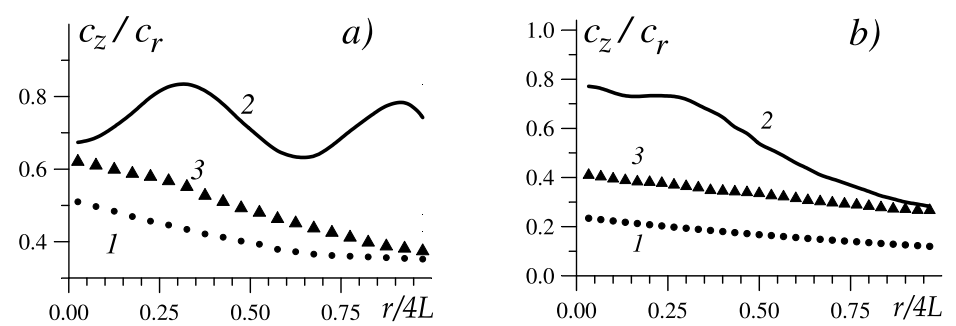

Fig. 8. Radial distributions of $\alpha_{z}=c_{z} / c_{r}$ for a) $\mu=1$ (see Fig. 1) and b) $\mu=4$ (see Fig. 5). The dotted curves (1) are for the initial distribution, solid lines (2) designate the final radial distributions and triangles (3) denote the critical levels of $\alpha_{z}$. 

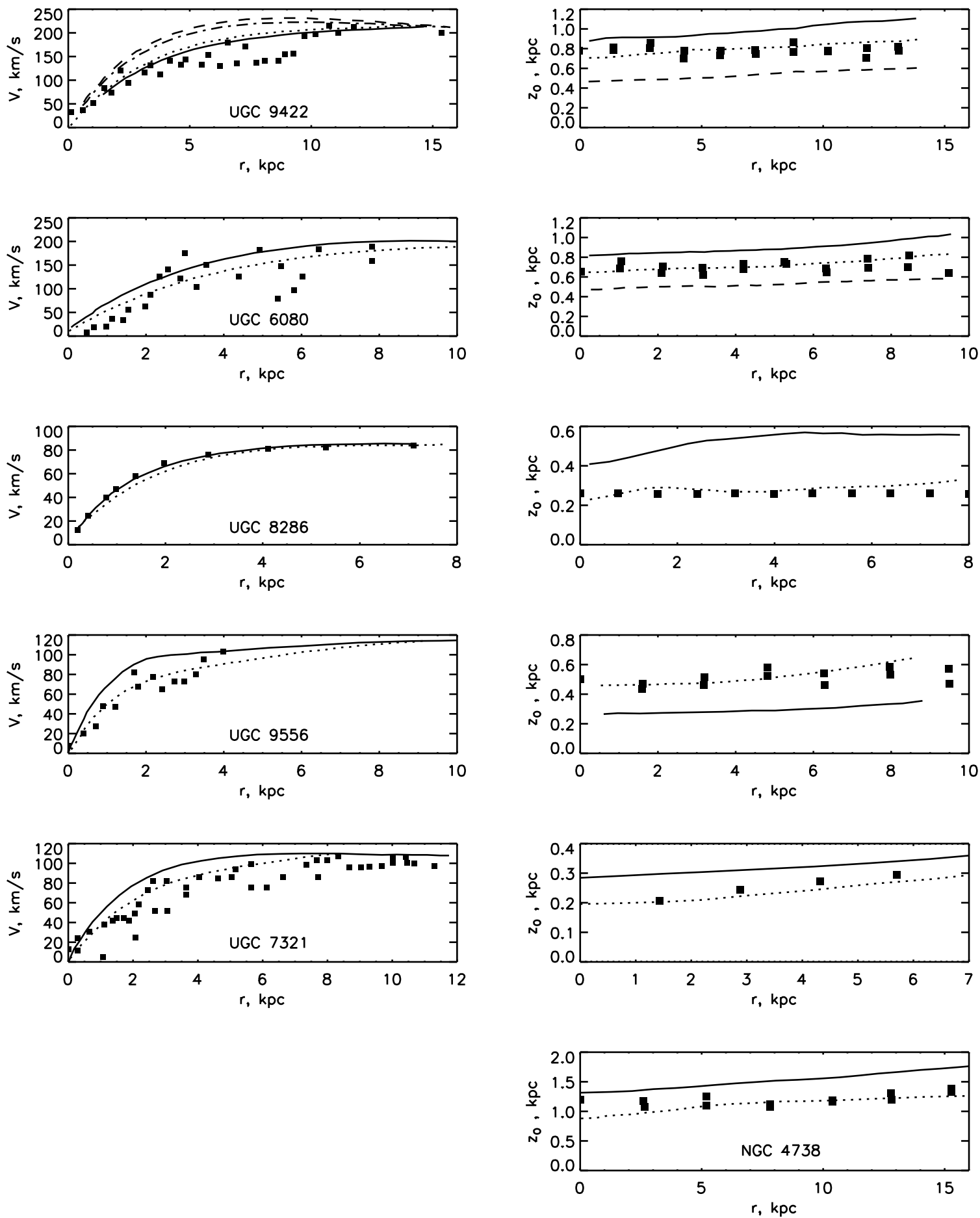

Fig. 9. Comparison of our modelling with observed values for UGC 9422, UGC 6080, UGC 8286, UGC 9556, UGC 7321 and NGC 4738 (from top to bottom). Left panels: radial distributions of $V_{c}$ (solid curves) and $V^{l o s}$, i.e. observed rotation curves in edge-on galaxies (dotted curves). Right panels: radial distributions of the projected scale height. Curves correspond to models with different $\mu$. See explanation of individual curves in \$4.1 Filled squares designate observed values (RC or disc thickness) in all panels. 


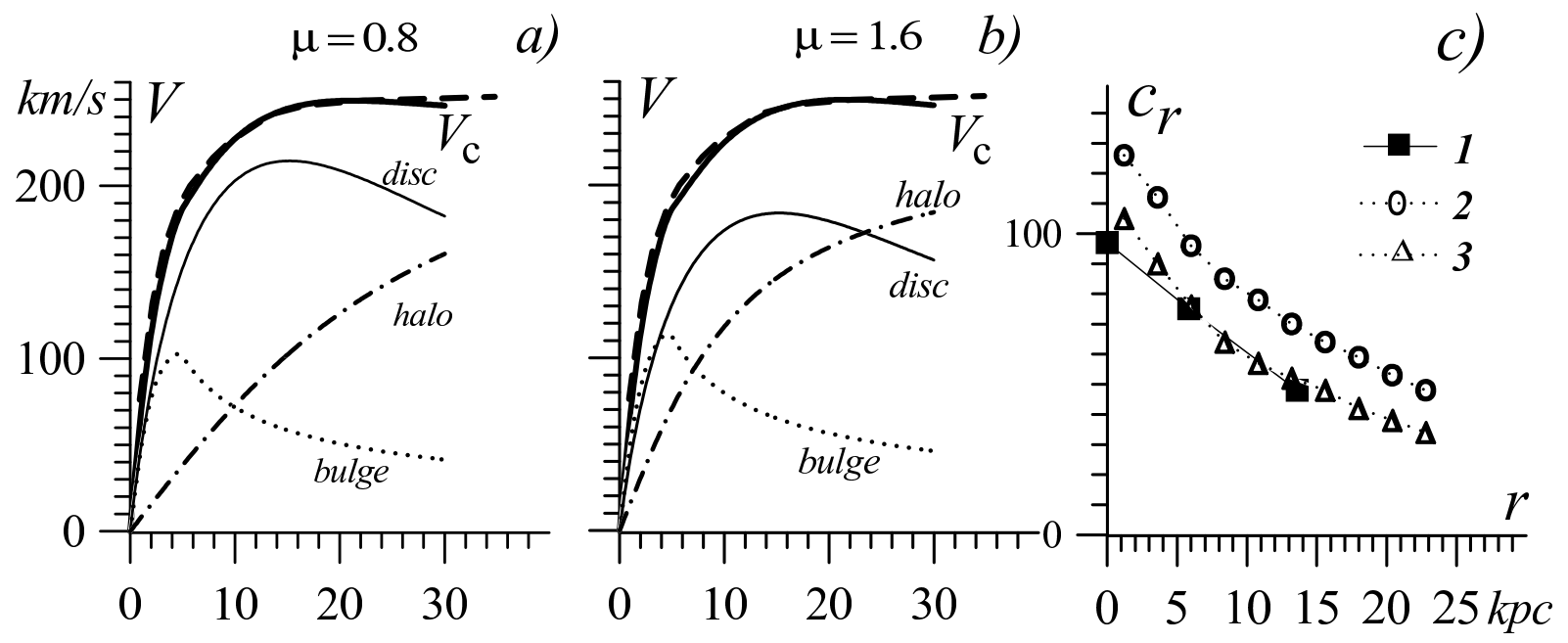

Fig. 10. Contributions of different galactic subsystems into the $V_{c}$ for two models of NGC 5170: $\mu=0.83$ (a) and $\mu=1.6$ (b). c) The radial velocity dispersions: 1 - from observations by Bottema et al. 1987, 2 - from the model with $\mu=0.83$ and $3-$ with $\mu=1.6$. 

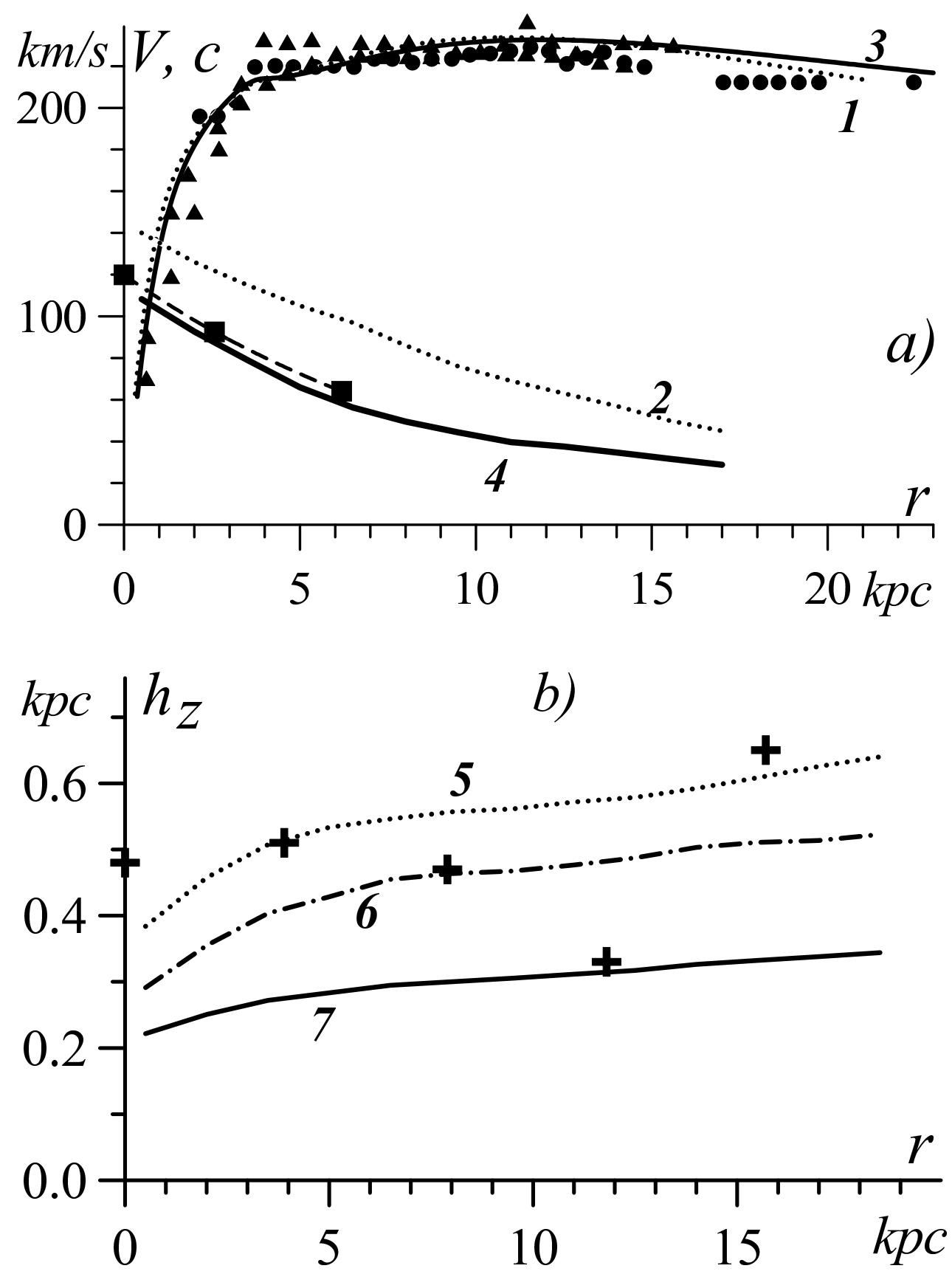

Fig. 11. NGC 891: a) Rotation curves from HI observations by Bottema et al. 1991 (circles) and Sancisi 1979 (triangles) and the velocity dispersion from Bottema et al. 1991, The model distributions of $V_{c}(1)$ and $c_{r}(2)$ are shown for the case of $M_{h} / M_{d}=0.46$, whereas the curves 3 and 4 correspond to $V_{c}$ and $c_{r}$, respectively, for the case of $M_{h} / M_{d}=1.5$. b) Observed (crosses) and model (lines) projected vertical scale height for the models with $M_{h} / M_{d}=0.4$ (the curve 5), 0.7 (6) and 1.7 (7). 


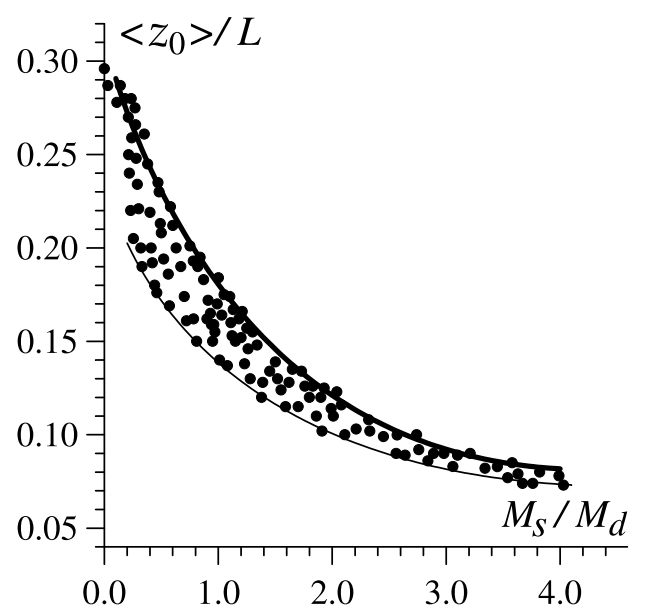

Fig. 12. The stellar disc thickness $\left(z_{0} / L\right)$ and the spherical-to-disc mass ratio $M_{s} / M_{d}$. The circles designate models on the stability boundary with different parameters of spherical subsystem and initial conditions in the modelling. The upper curve corresponds to bulgeless models whereas the lower one shows the models with noticeable bulges. 


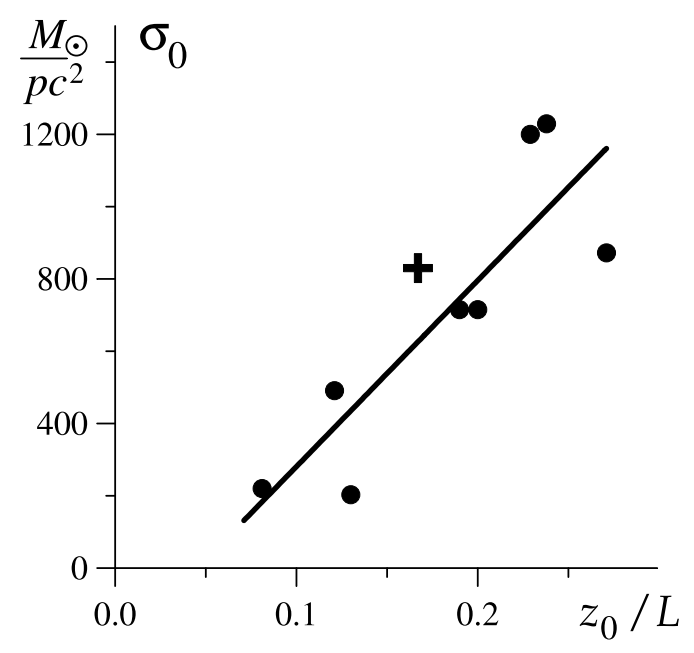

Fig. 13. The stellar disc thickness $\left(z_{0} / L\right)$ and the disc central surface density (in $M_{\odot} p c^{-2}$ ) for the galaxies from our sample (circles). The cross designates our Galaxy (Khoperskov \& Tyurina 2001) and the solid line is the least-square linear regression. 


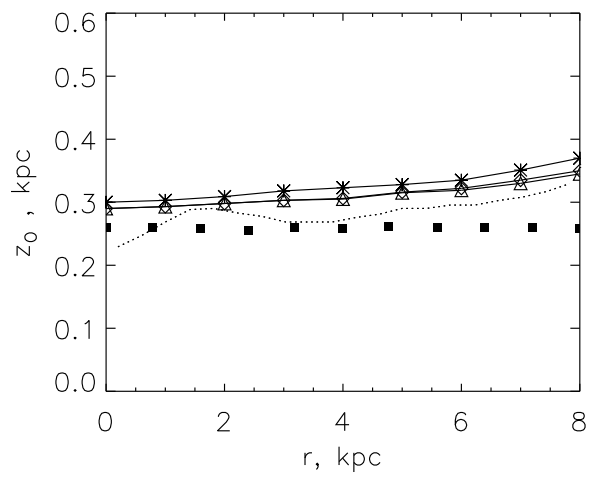

Fig. 14. Results of "live" halo modelling for one of our galaxies, UGC 8286. This figure is the same as the third upper and right panel in Figure 9 with addition of three models which are designated by the solid curves with asterisks, diamonds, and triangles, which correspond to $2 \cdot 10^{5}, 4 \cdot 10^{5}$ and $6 \cdot 10^{5}$ bodies in the "live" halo, respectively. 